\title{
MULTIFRACTAL FORMALISM FOR BENEDICKS-CARLESON QUADRATIC MAPS
}

\author{
YONG MOO CHUNG* AND HIROKI TAKAHASI**
}

\begin{abstract}
For a positive measure set of nonuniformly expanding quadratic maps on the interval we effect a multifractal formalism, i.e., decompose the phase space into level sets of time averages of a given continuous function and consider the associated Birkhoff spectrum which encodes this decomposition. We derive a formula which relates the Hausdorff dimension of level sets to entropies and Lyapunov exponents of invariant probability measures, and then use this formula to show that the spectrum is continuous. In order to estimate the Hausdorff dimension from above, one has to "see" sufficiently many points. To this end, we construct a family of towers. Using these towers we establish a large deviation principle of empirical distributions, with Lebesgue as a reference measure.
\end{abstract}

\section{INTRODUCTION}

Let $X=[-1,1]$, and let $f_{a}: X \circlearrowleft$ be the quadratic map given by $f_{a} x=1-a x^{2}$, where $0<a \leq 2$. It is well-known [2, 3, 17] that there exists a set of $a$-values near 2 with positive Lebesgue measure for which the corresponding $f=f_{a}$ admits an invariant probability measure $\mu$ that is absolutely continuous with respect to Lebesgue. In this paper we develop a theory of multifractal formalism for a positive measure set of these quadratic maps.

Given a function $\varphi: X \rightarrow \mathbb{R}$ we consider sets of the form

$$
K_{\varphi}(\alpha)=\left\{x \in X: \lim _{n \rightarrow \infty} \frac{1}{n} S_{n} \varphi(x)=\alpha\right\}, \quad \alpha \in \mathbb{R},
$$

where $S_{n} \varphi=\sum_{i=0}^{n-1} \varphi \circ f^{i}$. The following characteristic of the sets $K_{\varphi}(\alpha)$ has been studied in the literature:

$$
B_{\varphi}(\alpha)=\operatorname{dim}_{H} K_{\varphi}(\alpha),
$$

where $\operatorname{dim}_{H}$ denotes the Hausdorff dimension. This function of $\alpha$ is called a Birkhoff spectrum of $\varphi$. In the case $\varphi=\log |D f|$ it is called a Lyapunov spectrum. Multifractal formalism aims to relate these spectra to other characteristics of the system, and to study the regularity of the spectra as functions of $\alpha$, for instance, continuity, smoothness and convexity. With this study one tries to get more refined descriptions of the dynamics than purely stochastic considerations.

In the creation of the theory of multifractal formalism, uniform hyperbolicity or the absence of critical points have been assumed to obtain good descriptions of the spectra (see e.g. [9, 26, 28, 29, 30, 35]). Our aim here is to incorporate into the theory certain nonuniformly expanding quadratic maps on the interval with critical points. We provide a simple set of

2010 Mathematics Subject Classification. 37D25, 37D35, 37E05, 60F10.

*Department of Applied Mathematics, Hiroshima University, Higashi-Hiroshima 739-8527, JAPAN chung@amath.hiroshima-u.ac.jp **Department of Electronic Science and Engineering, Kyoto University, Kyoto 606-8501, JAPAN takahasi.hiroki.7r@kyoto-u.ac.jp. 
conditions satisfied on a positive measure set in the parameter space of the quadratic maps, and give a partial description of the Birkhoff spectrum when these conditions are met.

We formulate our conditions as follows:

(A1) $f=f_{a}$ where $a$ is sufficiently near 2 ;

(A2) $\left|D f^{n}(f 0)\right| \geq e^{\lambda n}$ for every $n \geq 0$, where $\lambda=\frac{9}{10} \log 2$;

(A3) $\left|f^{n} 0\right| \geq e^{-\frac{1}{100} \sqrt{n}}$ for every $n \geq 1$;

(A4) $f$ is topologically mixing on $\left[f^{2} 0, f 0\right]$.

Benedicks \& Carleson [3] proved the the abundance of parameters near 2 for which (A2) holds. For these parameters, there exists a unique absolutely continuous invariant probability measure $\mu$ (acip for short). The abundance of parameters for which (A3) holds was proved by Benedicks \& Young [4], and previously by Benedicks \& Carleson [2] under slightly different hypotheses. For their parameters, (A4) holds (see [37, Lemma 2.1]). The parameter sets they constructed have 2 as a full Lebesgue density point. Hence, given $a_{0}<2$ arbitrarily near 2 , there is a set $A \subset\left[a_{0}, 2\right]$ with positive Lebesgue measure such that (A2)-(A4) hold for all $a \in A$.

Let $C(X)$ denote the space of continuous functions on $X$, and $\mathcal{M}_{f}$ the space of $f$-invariant probability measures endowed with the topology of weak convergence. For $\varphi \in C(X)$ define

$$
c_{\varphi}=\inf _{x \in X} \frac{\lim }{n \rightarrow \infty} \frac{1}{n} S_{n} \varphi(x) \text { and } d_{\varphi}=\sup _{x \in X} \varlimsup_{n \rightarrow \infty} \frac{1}{n} S_{n} \varphi(x) .
$$

Since $\mathcal{M}_{f}$ is compact and $\varphi$ is continuous, one has $c_{\varphi}=\min \left\{\nu(\varphi): \nu \in \mathcal{M}_{f}\right\}$ and $d_{\varphi}=$ $\max \left\{\nu(\varphi): \nu \in \mathcal{M}_{f}\right\}$, where $\nu(\varphi)=\int \varphi d \nu$. Define sets $K_{\varphi}(\alpha)$ as above, and consider the decomposition

$$
X=\left(\bigcup_{\alpha \in\left[c_{\varphi}, d_{\varphi}\right]} K_{\varphi}(\alpha)\right) \cup \hat{K}_{\varphi}
$$

Here, $\hat{K}_{\varphi}$ is the set of points in $X$ for which $(1 / n) S_{n} \varphi$ does not converge. This decomposition has extremely complicated topological structures. Indeed, by $(\mathrm{A} 4), K_{\varphi}(\alpha)$ and $\hat{K}_{\varphi}$ are dense in $X$ unless they are empty. If $c_{\varphi}<d_{\varphi}$, then $\hat{K}_{\varphi}$ is nonempty and carries the full Hausdorff dimension [1, 6]. Since $c_{\varphi}$ and $d_{\varphi}$ are attained by ergodic measures, both $K_{\varphi}\left(c_{\varphi}\right)$ and $K_{\varphi}\left(d_{\varphi}\right)$ are nonempty. Using (A4) one can construct points with time averages converging to any number $\alpha \in\left(c_{\varphi}, d_{\varphi}\right)$. Hence any $K_{\varphi}(\alpha)$ in the decomposition is nonempty.

Let $h(\nu)$ denote the entropy of $\nu \in \mathcal{M}_{f}$ and define $\lambda(\nu)=\int \log |D f| d \nu$ which we call the Lyapunov exponent of $\nu$. This value is well-defined [5], and by a result of [25],

$$
\lambda_{\text {inf }}=\inf \left\{\lambda(\mu): \mu \in \mathcal{M}_{f}\right\}>0 .
$$

Relationships between entropies, Lyapunov exponents, and dimensions of invariant probability measures were studied in the literature [15, 22, 36]. The next theorem relates the Birkhoff spectrum to entropies and Lyapunov exponents of invariant probability measures.

Theorem A. If $f=f_{a}$ satisfies (A1)-(A4), then for any $\varphi \in C(X)$ and $\alpha \in\left[c_{\varphi}, d_{\varphi}\right]$,

$$
B_{\varphi}(\alpha)=\lim _{\varepsilon \rightarrow 0} \sup \left\{\frac{h(\nu)}{\lambda(\nu)}: \nu \in \mathcal{M}_{f},|\nu(\varphi)-\alpha|<\varepsilon\right\} .
$$

In addition, the Birkhoff spectrum $\alpha \mapsto B_{\varphi}(\alpha)$ is continuous. 
In [6], the first-named author derived the same formula as in Theorem A for a class of one-dimensional maps. This class includes maps whose critical points are non-recurrent and with no neutral or stable periodic point (the so-called Misiurewicz maps). Theorem A allows the recurrence of the critical point at a sub-exponential rate by condition (A3). Although $\varphi$ is required to be continuous, an extension of the formula to cover the Lyapunov spectrum will be given in our forthcoming work.

The multifractal formalism for one-dimensional maps with critical points is a rapidly expanding area of research, and quite a few results have been obtained lately. For multimodal maps satisfying growth conditions of derivatives along the orbits of critical points, Iommi \& Todd [16] obtained a formula which relates the Lyapunov spectra to thermodynamic pressures. See Gelfert, Przytycki \& Rams [12] and Przytycki \& Rivera-Letelier [31] for results on the Lyapunov spectra of rational maps on the Riemannian sphere. A key idea common to these recent works is to construct a sequence of nice induced systems that "exhausts" the original system. Although a proof of Theorem A relies on the same idea, our induced systems are equipped with a special recurrence property. This requires a new construction.

The formula in Theorem A yields several properties of the Birkhoff spectrum. For instance, it is easy to show that $B_{\varphi}$ is monotone increasing on the interval $\left[c_{\varphi}, \mu(\varphi)\right]$, while it is monotone decreasing on $\left[\mu(\varphi), d_{\varphi}\right]$ as a function of $\alpha$. From the formula it readily follows that $B_{\varphi}$ is upper semi-continuous. We are able to show that $B_{\varphi}$ is lower semi-continuous, and so it is continuous. This phenomenon illustrates what is sometimes called the multifractal miracle even though the decomposition of the phase space into the level sets is intricate and extremely complicated, the function $B_{\varphi}$ which encodes this decomposition is continuous.

It is an interesting problem to study better regularities of the spectrum. If the dynamics is uniformly hyperbolic and the function $\varphi$ is Hölder continuous, then the spectrum is real analytic and concave [30]. For one-dimensional maps with parabolic fixed points, the nonanalyticity of the Lyapunov spectra implies the finiteness of absolutely continuous invariant measures [24]. For the quadratic maps, only numerical results are known (see e.g. [13, 14]).

Our strategy for the lower estimate of $B_{\varphi}(\alpha)$ is to construct certain Cantor sets in $K_{\varphi}(\alpha)$, and then put probability measures on them for which the Mass Distribution Principle holds (see [36, Proposition 2.1]). The presence of the critical point does not matter because small derivatives tend to improve lower estimates of Hausdorff dimension.

For the upper estimate, we approximate $B_{\varphi}(\alpha)$ from above by the dimensions of ergodic measures (cf. Proposition 3.1). To construct such an ergodic sequence we construct a family of uniformly hyperbolic induced systems with finitely many branches. We pick the corresponding family of equilibrium states for weighted geometric potentials, and then spread them out to produce a sequence of ergodic measures with the desired property.

The biggest difficulty is to construct such a family of induced systems. We do this in two steps. We first construct a family of towers, with a special property that a positive definite fraction of points in each partition element quickly fall down to the ground floor. For this construction we make an important use of condition (A3). We then construct the desired family of induced systems by choosing a subsystem from each tower dynamical system.

Using the family of towers used in the proof of Theorem A we establish a large deviation principle for the Lebesgue measure. Let $\mathcal{M}$ denote the space of probability measures on $X$ endowed with the topology of weak convergence. Define a free energy function $F: \mathcal{M} \rightarrow$ 
$\mathbb{R} \cup\{-\infty\}$ by

$$
F(\nu)= \begin{cases}h(\nu)-\lambda(\nu) & \text { if } \nu \in \mathcal{M}_{f} \\ -\infty & \text { otherwise. }\end{cases}
$$

By Ruelle's inequality [33], $F(\nu) \leq 0$ and the equality holds only if $\nu=\mu$ [21].

It is known [5] that the Lyapunov exponent is not lower semi-continuous, and so $-F$ may not be lower semi-continuous. Hence we introduce its lower-semi-continuous regularization $I: \mathcal{M} \rightarrow[0, \infty]$ by

$$
I(\nu)=-\inf _{\mathcal{G}} \sup \{F(\xi): \xi \in \mathcal{G}\},
$$

where the infimum is taken over all neighborhoods $\mathcal{G}$ of $\nu$ in $\mathcal{M}$. Denote by $|\cdot|$ the Lebesgue measure on $X$ and let $\log 0=-\infty$. Let $\delta_{x}^{n}=(1 / n) \sum_{i=0}^{n-1} \delta_{f^{i} x}$ where $\delta_{f^{i} x}$ is the Dirac measure at $f^{i} x$.

Theorem B. Let $f=f_{a}$ satisfy (A1)-(A4). Then the large deviation principle holds for $(f,|\cdot|)$ with $I$ the rate function, namely for any open set $\mathcal{G} \subset \mathcal{M}$,

$$
\varliminf_{n \rightarrow \infty} \frac{1}{n} \log \left|\left\{x \in X: \delta_{x}^{n} \in \mathcal{G}\right\}\right| \geq-\inf \{I(\nu): \mu \in \mathcal{G}\},
$$

and for any closed set $\mathcal{K} \subset \mathcal{M}$,

$$
\varlimsup_{n \rightarrow \infty} \frac{1}{n} \log \left|\left\{x \in X: \delta_{x}^{n} \in \mathcal{K}\right\}\right| \leq-\inf \{I(\nu): \mu \in \mathcal{K}\} .
$$

The large deviation principle has been proved in different settings, for different reference measures and with different assumptions on the hyperbolicity of the systems [10, 20, 27, 31. For a positive measure set of quadratic maps we treat here, the large deviation principle for the acips was proved in [8]. Theorem B is not a consequence of this, because the density of the acip is unbounded.

The Contraction Principle in large deviations [11] allows us to obtain a formula for fluctuations of time averages of continuous functions. Let $\varphi \in C(X)$. We assume $c_{\varphi}<d_{\varphi}$, for otherwise it is meaningless to consider $\varphi$. Define a function $F_{\varphi}:\left[c_{\varphi}, d_{\varphi}\right] \rightarrow \mathbb{R}$ by

$$
F_{\varphi}(\alpha)=\sup \left\{F(\nu): \nu \in \mathcal{M}_{f}, \nu(\varphi)=\alpha\right\} .
$$

Corollary 1. If $c_{\varphi} \leq \alpha<\beta \leq d_{\varphi}$, then

$$
\lim _{n \rightarrow \infty} \frac{1}{n} \log \left|\left\{\alpha \leq \frac{1}{n} S_{n} \varphi \leq \beta\right\}\right|=\max _{\alpha \leq t \leq \beta} F_{\varphi}(t) .
$$

Keller \& Nowicki [19] obtained a local result which claims the existence of the limit provided $\varphi$ is Hölder continuous and $\alpha, \beta$ are sufficiently near the mean $\mu(\varphi)$. Corollary 1 is a full result with no restriction on $\alpha$ or $\beta$.

The next corollary follows from Varadhan's integral lemma [11, p.137] and the convex duality of Fenchel-Legendre transforms [11, p.152].

Corollary 2. For any $\varphi \in C(X)$, the limit

$$
P(\varphi)=\lim _{n \rightarrow \infty} \frac{1}{n} \log \int e^{S_{n} \varphi} d x
$$

exists. In addition, $(P, I)$ form a Legendre pair, namely the following holds:

$$
P(\varphi)=\max \left\{\nu(\varphi)-I(\nu): \nu \in \mathcal{M}_{f}\right\} \quad \text { for all } \varphi \in C(X) ;
$$




$$
I(\nu)=\max \{\nu(\varphi)-P(\varphi): \varphi \in C(X)\} \quad \text { for all } \nu \in \mathcal{M}_{f}
$$

The rest of this paper consists of four sections. In Sect.2 we construct a family of towers, collecting materials in [8] as far as needed. In Sect.3, using this family of towers we estimate $B_{\varphi}(\alpha)$ from above. In Sect.4 we estimate $B_{\varphi}(\alpha)$ from below and complete the proof of the formula in Theorem A. We then use this formula to prove the continuity of the Birkhoff spectrum. In Sect.5 we prove Theorem B.

\section{Construction of a FAmily of tOWERS}

In this section, for a map $f$ satisfying (A1)-(A4) we first introduce the machinery in [8] for recovering small derivatives near the critical point. We then construct a family of induced maps and associated towers. Important constants are $0<\varepsilon \ll 1$ and $N \gg 1$, chosen in this order. In this section we suppose they are given. In Sect. 3 and Sect.5 we let $\varepsilon$ approach 0 .

We use the following standard notation: for a set $A \subset X, d(0, A)=\inf \{|x|: x \in A\}$; given a partition $\mathcal{P}$ of $A \subset X$ and $B \subset A, \mathcal{P} \mid B=\{\omega \cap B: \omega \in \mathcal{P}\}$.

2.1. Recovering expansion. The next lemma states that the dynamics outside of a small neighborhood of the critical point is uniformly expanding with an exponent independent of the size of the neighborhood.

Lemma 2.1. (cf. [8, Lemma 2.5]) The following holds for any $\hat{\delta}>0$ : if $x \in X, n \geq 1$ are such that $\left|f^{i} x\right| \geq \hat{\delta}$ for every $0 \leq i \leq n-1$, then $\left|D f^{n}(x)\right| \geq \hat{\delta} e^{\frac{\lambda}{3} n}$. Moreover, if $\left|f^{n} x\right|<\hat{\delta}$, then $\left|D f^{n}(x)\right| \geq e^{\frac{\lambda}{3} n}$.

A proof of this lemma is almost identical to that of [8, Lemma 2.5], and hence is omitted. Although particular values of $\hat{\delta}$ were chosen there, this choice is not essential.

To deal with the loss of expansion due to returns to the critical region we mimic the binding argument of Benedicks \& Carleson [2, 3]: subdivide the interval into pieces, and deal with them independently. For $p>0$ let

$$
\delta_{p}=\sqrt{\frac{e^{-\varepsilon p}}{10}\left[\sum_{i=0}^{p-1} \frac{\left|D f^{i}(f 0)\right|}{\left|f^{i+1} 0\right|}\right]^{-1}} .
$$

If $\delta_{p} \leq|x|<\delta_{p-1}$, then we regard the orbit of $x$ as bound to the orbit of 0 up to time $p$.

Lemma 2.2. For any $\varepsilon>0$ there exists $N>0$ such that if $p \geq N$ and $\delta_{p} \leq|x|<\delta_{p-1}$, then:

(a) $\left|D f^{p}(x)\right| \geq e^{\frac{\lambda}{3} p}$

(b) $\log |x|^{-\frac{2}{\log 5}} \leq p \leq \log |x|^{-\frac{2}{\lambda}}$.

Proof. (a) and the second inequality in (b) are due to [8, Lemma 2.3]. Rearranging $|x|^{2} \geq$ $\delta_{p}^{2} \geq 5^{-p}$ which follows from the definition of $\delta_{p}$ in (1), and then using $|D f| \leq 4$, (A3) yield the first inequality in (b).

2.2. Construction of a partition with slowly recurrent points. We construct a partition of a small neighborhood of the critical point which is well-adapted to later constructions.

To start, for each $p>N$ cut the interval $\left[\delta_{p}, \delta_{p-1}\right)$ into $\left[e^{3 \varepsilon p}\right]$-number of intervals of equal length and denote them by $\hat{I}_{p, j}\left(j=1,2, \ldots,\left[e^{3 \varepsilon p}\right]\right)$, from the right to the left. This defines a partition of the interval $\left(0, \delta_{N}\right)$, but it is not satisfactory for our construction, because there is 
no control over the iterates of the boundary points of the partition elements. To rectify this, we show in the next lemma the existence of a point in each $\hat{I}_{p, j}$ which is slowly recurrent to the critical point 0 . We then use these points as partition points.

Lemma 2.3. For each $(p, j)$ there exists $x \in \hat{I}_{p, j}$ such that $\left|f^{n} x\right| \geq \delta_{N} e^{-\varepsilon n}$ for every $n \geq \varepsilon^{-1}$. Proof. Set $t_{0}=0, \omega_{0}=\hat{I}_{p, j}$ and $p_{0}=p$. For every $\varepsilon^{-1} \leq n \leq p_{0}$ we have

$$
d\left(0, f^{n} \omega_{0}\right) \geq\left|f^{n} 0\right|-\left|f^{n} \omega_{0}\right| \geq(1 / 2)\left|f^{n} 0\right| \geq(1 / 2) e^{-\frac{\sqrt{n}}{100}} \geq e^{-\varepsilon n},
$$

where we have used the bounded distortion of $f^{n-1}$ on $f \omega_{0}$ from [8, Lemma 2.1] for the second inequality. The third one follows from (A3). The last one holds for sufficiently small $\varepsilon$.

By induction we choose a sequence $n_{0}<n_{1}<\cdots$ of integers and a sequence $\omega_{0} \supsetneq \omega_{1} \supsetneq \cdots$ of closed intervals such that for every $k \geq 0$,

(3) $f^{n_{k}} \omega_{k}=\hat{I}_{p_{k}, j_{k}}$ for some $p_{k}, j_{k}$ and $d\left(0, f^{n} \omega_{k}\right) \geq \delta_{N} e^{-\varepsilon n}$ for every $n_{k} \leq n \leq n_{k}+p_{k}-1$.

From (2) and (3) , the point in the singleton $\bigcap_{k>0} \omega_{k}$ satisfies the desired property.

For the rest of the proof, we assume (3) holds for some $k=l$, and then indicate how to choose $t_{l+1}$ and $\omega_{l+1}$ for which (3) holds for $k=l+1$. An argument to show (3) for $k=0$ is included in the general step of the induction below.

Given $n_{l}, \omega_{l}$ such that $f^{n_{l}} \omega_{l}=\hat{I}_{p_{l}, j_{l}}$, define $n_{l}+p_{l} \leq t_{1}<t_{2}<\cdots$ inductively as follows: $t_{1}$ is the smallest $t \geq n_{l}+p_{l}$ with $d\left(0, f^{t} \omega_{l}\right)<\delta_{N}$. Suppose $t_{i}$ has been defined. If $f^{t_{i}} \omega_{l}$ intersects no more than two $\hat{I}_{p, j^{-}}$intervals, say $\hat{I}_{p, j}$ and $\hat{I}_{p^{\prime}, j^{\prime}}, p \leq p^{\prime}$ (possibly $\hat{I}_{p, j}=\hat{I}_{p^{\prime}, j^{\prime}}$ ), then define $t_{i+1}$ to be the smallest $t \geq t_{i}+q_{i}$ with $d\left(0, f^{t} \omega_{l}\right)<\delta_{N}$, where $q_{i}=p$ if $f^{t_{i}} \omega_{l} \subset\left(-\delta_{N}, \delta_{N}\right)$, and $q_{i}=1$ otherwise. If $f^{t_{i}} \omega_{l}$ intersects more than three $\hat{I}_{p, j}$-intervals, then $t_{i+1}$ is undefined.

The expansion estimates in Lemma 2.1 and Lemma 2.2 imply that one finally reaches $t_{s}$ such that $f^{t_{s}} \omega_{l}$ intersects more than three $\hat{I}_{p, j}$-intervals. For all $\theta \in f^{n_{l}+p_{l}} \omega_{l}$ we have

$$
\left|D f^{n_{l+1}-n_{l}-p_{l}}(\theta)\right| \geq \delta \exp \left(\frac{\lambda}{3} \sum_{\substack{1 \leq i \leq s-1 \\ q_{i} \neq 1}} q_{i}\right) \geq \delta_{N} .
$$

This and $\left|f^{n_{l}+p_{l}} \omega_{l}\right| \geq e^{-5 \varepsilon p_{l}}$ which follows from [8, Lemma 2.6(a)] yield $\left|f^{n_{l+1}} \omega_{l}\right| \geq \delta_{N} e^{-5 \varepsilon p_{l}}$. By Lemma $2.2(\mathrm{~b}), p_{l} \leq(2 / \lambda)\left(-\log \delta_{N}+\varepsilon n_{l}\right) \leq(3 / \lambda) \varepsilon n_{l}$, and thus $\left|f^{n_{l+1}} \omega_{l}\right| \geq \delta_{N} e^{-5 \varepsilon p_{l}} \geq$ $\delta_{N} e^{-\frac{15}{\lambda} \varepsilon^{2} n_{l+1}}$. From this and the upper estimate of the length of $\hat{I}_{p, j}$ in [8, Lemma 2.6(b)], one can choose $\omega_{l+1} \subset \omega_{l}$ such that $f^{n_{l+1}} \omega_{l+1}=\hat{I}_{p_{l+1}, j_{l+1}}$ and $d\left(0, f^{n_{l+1}} \omega_{l+1}\right) \geq \delta_{N} e^{-\varepsilon n_{l+1}}$.

It is left to estimate the distance of the forward iterates of $f^{n_{l}+p_{l}-1} \omega_{l+1}$ to the critical point. We first consider the case $n=t_{i}$ with $q_{i} \neq 1$. (44) implies that for some $\theta \in f^{n_{l}+p_{l}} \omega_{l}$ we have

$$
2 \geq\left|f^{n_{l+1}} \omega_{l}\right|=\left|D f^{n_{l+1}-n_{l}-p_{l}}(\theta)\right| \cdot\left|f^{n_{l}+p_{l}} \omega_{l}\right| \geq \delta_{N} e^{\frac{\lambda}{3} q_{i}} e^{-5 \varepsilon p_{l}} .
$$

Taking logs and then rearranging the result we have

$$
q_{i} \leq-(4 / \lambda) \log \delta_{N}+(15 / \lambda) \varepsilon p_{l} \leq(16 / \lambda) \varepsilon p_{l} \leq\left(48 / \lambda^{2}\right) \varepsilon^{2} n_{l} .
$$

Hence, for $n=t_{i}$ we obtain

$$
d\left(0, f^{n} \omega_{l}\right) \geq \delta_{q_{i}} \geq 5^{-q_{i}} \geq e^{-\varepsilon n_{l}} \geq \delta_{N} e^{-\varepsilon n} .
$$


Next we consider the case $n \in\left(t_{i}, t_{i}+q_{i}\right]$ with $q_{i} \neq 1$. Let $J$ denote the minimal interval containing $f^{t_{i}} \omega_{l}$ and 0 . Then $|f J| \leq 2 \delta_{q_{i}}^{2}$, and the distortion of $f^{n-t_{i}-1}$ on $f J$ is bounded by [8, Lemma 2.1]. Hence

$$
\left|f^{n-t_{i}} J\right| \leq 2\left|D f^{n-t_{i}-1}(f 0)\right||f J| \leq 4\left|D f^{n-t_{i}-1}(f 0)\right| \delta_{q_{i}}^{2} \leq(9 / 10)\left|f^{n-t_{i}+1} 0\right|,
$$

and thus

$$
d\left(0, f^{n} \omega_{l}\right) \geq\left|f^{n-t_{i}+1} 0\right|-\left|f^{n-t_{i}} J\right| \geq(1 / 10)\left|f^{n-t_{i}+1} 0\right| \geq(1 / 10) e^{-\frac{1}{100} \sqrt{q_{i}}} \geq e^{-\varepsilon t_{i}} \geq \delta e^{-\varepsilon n} .
$$

The second inequality follows from (A3) and the third from (5).

Note that the above argument may be extended to the case $n \in\left[n_{l+1}+1, n_{l+1}+p_{l+1}-1\right]$. For all $n \in\left[n_{l}+p_{l}-1, n_{l+1}-1\right]$ other than those treated so far, the desired estimate holds because $f^{n} \omega_{l}$ is not contained in $\left(-\delta_{N}, \delta_{N}\right)$ and intersects at most one $\hat{I}_{p, j}$. The assumption of the induction has been recovered.

In view of Lemma 2.3, for each $\hat{I}_{p, j}$ fix once and for all a point $x_{p, j} \in \hat{I}_{p, j}$ such that $\left|f^{n} x_{p, j}\right| \geq \delta_{N} e^{-\varepsilon n}$ holds for every $n \geq \varepsilon^{-1}$. Set $\delta=x_{N, 1}$. Note that $\delta<\delta_{N}$, and $\delta \rightarrow 0$ as $N \rightarrow \infty$. Using the points $x_{p, j}$ as partition points we construct a countable partition of the interval $(0, \delta)$ in such a way that: 1

(i) each element of the partition contains exactly one element of $\left\{\hat{I}_{p, j}\right\}$;

(ii) each element of the partition is contained in three contiguous elements of $\left\{\hat{I}_{p, j}\right\}$.

The construction is straightforward. The boundary points of partition elements belong to $\left\{x_{p, j}\right\}$. Let $I_{p, j}$ denote the element of the partition containing $\hat{I}_{p, j}$. Let $I_{p,-j}=-I_{p, j}$, the mirror image of $I_{p, j}$ with respect to 0 .

2.3. Construction of dynamical partitions. Let $\Lambda^{+}=I_{N, 1}$, which is the right extremal $I_{p, j}$-interval. Let $\Lambda^{-}=-\Lambda^{+}$and $\Lambda=\Lambda^{-} \cup \Lambda^{+}$. Let $\hat{x}$ denote the orientation reversing fixed point of $f$ in $X$ and set $\hat{X}=[-\hat{x}, \hat{x}]$. By induction on the number of iterations we construct a "decreasing" sequence $\left\{\tilde{\mathcal{P}}_{n}\right\}_{n \geq 0}$ of partitions of $\hat{X}$ into intervals, and introduce the notion of bound/free states.

Start with $\tilde{\mathcal{P}}_{0}=\{[-\hat{x},-\delta],[\delta, \hat{x}]\} \cup\left\{I_{p, j}\right\}_{p, j}$. We refer to the intervals $f[-\hat{x},-\delta], f[\delta, \hat{x}]$, $f I_{p, j}$ and $f^{p} I_{p, j}$ as free, and to $f^{i} I_{p, j}(1 \leq i \leq p-1)$ as bound. Call $p$ a bound period of $I_{p, j}$ at time 0 .

Let $n \geq 1$. The $f^{n}$-images of elements of $\tilde{\mathcal{P}}_{n-1}$ are in two phases: either bound or free. If $\omega \in \tilde{\mathcal{P}}_{n-1}, f^{n} \omega$ is free and $d\left(0, f^{n} \omega\right)<\delta$, then $\tilde{\mathcal{P}}_{n}$ subdivides $\omega$. For each resulting element $\omega^{\prime} \in \tilde{\mathcal{P}}_{n} \mid \omega$ with $d\left(0, f^{n} \omega^{\prime}\right)<\delta$ an integer $p_{n}\left(\omega^{\prime}\right)$ is attached; this integer is called a bound period of $\omega^{\prime}$ at time $n$.

Given $\omega \in \tilde{\mathcal{P}}_{n-1}, \tilde{\mathcal{P}}_{n} \mid \omega$ is defined as follows. If $f^{n} \omega$ is free and contains more than two $I_{p, j}$-intervals, then let $\tilde{\mathcal{P}}_{n}$ subdivide $\omega$ according to the $(p, j)$-locations of its $f^{n}$-image. In all other cases, let $\tilde{\mathcal{P}}_{n} \mid \omega=\{\omega\}$. Partition points are inserted only to ensure that the $f^{n}$-images of $\tilde{\mathcal{P}}_{n}$-elements intersecting $(-\delta, \delta)$ contain exactly one $I_{p, j}$. The $f^{n}$-images out of $(-\delta, \delta)$ are treated as follows. Let $\omega^{\prime} \subset \omega$ be such that $f^{n} \omega^{\prime}$ is a component of $f^{n} \omega \backslash(-\delta, \delta)$. We let $\omega^{\prime} \in \tilde{\mathcal{P}}_{n}$ if $\left|f^{n} \omega^{\prime}\right| \geq\left|\Lambda^{+}\right|$. Otherwise, we glue $\omega^{\prime}$ to the adjacent element whose $f^{n}$-image is contained in $\Lambda^{ \pm}$.

\footnotetext{
${ }^{1}$ Note that not all $x_{p, j}$ are used in this construction, because of the requirement (ii).
} 
The bound periods at time $n$ of the elements of $\tilde{\mathcal{P}}_{n} \mid \omega$ are determined by the $p$-locations of their $f^{n}$-images. Namely, if $\tilde{\mathcal{P}}_{n}$ subdivides $\omega$ and $\omega^{\prime} \in \tilde{\mathcal{P}}_{n} \mid \omega$, then $p_{n}\left(\omega^{\prime}\right)=p$ where $p$ is such that $f^{n} \omega^{\prime} \supset I_{p, j}$ holds for some $j$. If $\tilde{\mathcal{P}}_{n} \mid \omega=\{\omega\}$, then $p_{n}(\omega)=\min \left\{p: I_{p, j} \cap f^{n} \omega \neq\right.$ $\emptyset$ for some $j$ \}.

To proceed, for $\omega^{\prime} \in \tilde{\mathcal{P}}_{n}$ we say $f^{n+1} \omega^{\prime}$ is bound if there exists $k \leq n$ such that $\omega^{\prime} \in \tilde{\mathcal{P}}_{k}$, $p_{k}\left(\omega^{\prime}\right)$ makes sense and satisfies $n+1<k+p_{k}\left(\omega^{\prime}\right)$. Otherwise we say $f^{n+1} \omega^{\prime}$ is free. This completes the construction of $\tilde{\mathcal{P}}_{n}(n=0,1, \ldots)$.

The following bounded distortion can be proved similarly 2 to [8, Lemma 2.7]. Set $C_{0}=$ $\exp \left(-\delta^{3}\right)$. Let $\omega \in \tilde{\mathcal{P}}_{n-1}$ and suppose that $f^{n} \omega$ is free. Then

$$
\frac{\left|D f^{n}(x)\right|}{\left|D f^{n}(y)\right|} \leq C_{0} \quad \forall x, y \in \omega .
$$

2.4. Inducing time estimates. We define inductively a partition $\mathcal{Q}$ of $\Lambda$ into intervals and an associated inducing time $R: \mathcal{Q} \rightarrow \mathbb{N}$ as follows. Let $\omega \in \tilde{\mathcal{P}}_{n-1} \mid \Lambda$. If $f^{n} \omega$ is free and $f^{n} \omega \supset 3 \Lambda^{+}$or $3 \Lambda^{-}$, then set $\omega \cap f^{-n} \Lambda^{+} \in \mathcal{Q}$ or $\omega \cap f^{-n} \Lambda^{-} \in \mathcal{Q}$, and $R(\omega)=n$. We iterate the remaining parts $f^{n} \omega \backslash \Lambda^{+}$or $f^{n} \omega \backslash \Lambda^{-}$, which is the union of elements of $\tilde{\mathcal{P}}_{n}$, and repeat the same procedure. By definition, for each $\omega \in \mathcal{Q}, f^{R(\omega)}$ sends $\omega$ diffeomorphically onto $\Lambda^{+}$ or $\Lambda^{-}$.

Set $\hat{\zeta}=\left|\Lambda^{+}\right| /\left(2 C_{0}\right) \in(0,1)$ and $C_{1}=1+\hat{\zeta}^{-1}$. Note that $\hat{\zeta} \rightarrow 0$ and $C_{1} \rightarrow \infty$ as $\delta \rightarrow 0$. Let $\theta=10^{-10000}$ and set $\zeta=\max \left\{e^{-\frac{\lambda}{14}},(1-\hat{\zeta})^{\theta}\right\}$. Lemma 2.4 below applied to $\Lambda^{ \pm}$implies that the measure of the tail set

$$
\{R>n\}=\bigcup_{\omega \in \mathcal{Q}: R(\omega)>n} \omega
$$

decays exponentially fast. In particular, $\mathcal{Q}$ is a partition of a full measure subset of $\Lambda$.

Lemma 2.4. There exists $k_{0}=k_{0}(\delta)$ such that the following holds for every $k \geq k_{0}$ : let $\omega \in \tilde{\mathcal{P}}_{k-1}$ and suppose that $\omega \subset\{R>k\}$ and $f^{k} \omega$ is free. Then

$$
|\{R>k+l\} \cap \omega| \leq C_{1} \zeta^{l}|\omega| \text { for every } l \geq(16 \varepsilon / \lambda) k .
$$

Proof. Let $\mathcal{Q}^{\prime}$ denote the set of all $\omega^{\prime} \in \mathcal{Q} \mid\{R>k+l\} \cap \omega$ for which there exists $n \in[k, k+l]$ such that $d\left(0, f^{n} \omega_{n}\right)<\delta$ holds for the element $\omega_{n} \in \tilde{\mathcal{P}}_{n}$ containing $\omega^{\prime}$. Let $\mathcal{Q}^{\prime \prime}$ denote the collection of elements of $\mathcal{Q} \mid\{R>k+l\} \cap \omega$ which do not belong to $\mathcal{Q}^{\prime}$.

Each $\eta \in \mathcal{Q}^{\prime}$ has an itinerary $\left(n_{1}, p_{1}, j_{1}\right), \ldots,\left(n_{s}, p_{s}, j_{s}\right)(s \leq[l / N])$ that is defined as follows: $k \leq n_{1}<\cdots<n_{s} \leq k+l$ is a sequence of integers, associated with a sequence $\omega_{0} \supset \omega_{n_{1}} \supset \cdots \supset \omega_{n_{s}} \supset \eta$ of intervals such that $\omega_{n_{i}}(i=1, \ldots, s)$ is the element of $\tilde{\mathcal{P}}_{n_{i}}$ containing $\omega$ that arises out of the subdivision at time $n_{i}$, with $I_{p_{i}, j_{i}} \subset f^{n_{i}} \omega_{n_{i}} ; \omega_{n_{s}} \in \tilde{\mathcal{P}}_{k+l-1}$. Since $\left|f^{n_{s}+p_{s}} \omega_{n_{s}}\right| \leq 2$, for some $x \in \omega_{n_{s}}$ we have $\left|\omega_{n_{s}}\right| \leq 2\left|D f^{n_{s}+p_{s}}(x)\right|^{-1}$. By Lemma 2.1 and Lemma $2.2\left(\right.$ a) we have $\left|D f^{n_{s}+p_{s}}(x)\right| \geq \delta e^{\frac{\lambda}{3} \sum_{i=1}^{s} p_{i}}\left|D f^{k}(x)\right|$, and by (6) $),\left|D f^{k}(x)\right| \geq$ $\left(1 / C_{0}\right)\left|f^{k} \omega\right| /|\omega|$. Combining these three inequalities we obtain

$$
|\eta| \leq\left|\omega_{n_{s}}\right| \leq 2 C_{0} \delta^{-1} e^{-\frac{\lambda}{3} \sum_{i=1}^{s} p_{i}} \frac{|\omega|}{\left|f^{k} \omega\right|},
$$

\footnotetext{
${ }^{2}$ Although the value of " $\delta$ " is slightly different from the one used in $[8$, the technical adjustment is minimal.
} 
and therefore

$$
\sum_{\eta \in \mathcal{Q}^{\prime}}|\eta| \leq 2 C_{0} \delta^{-1} \sum_{s} \sum_{P} e^{-\frac{\lambda}{3} P} \#\left\{\left\{\left(n_{i}, p_{i}, j_{i}\right)\right\}_{i=1}^{s}: \sum_{i=1}^{s} p_{i}=P\right\} \frac{|\omega|}{\left|f^{k} \omega\right|}
$$

From the proof of [8, Lemma 2.8] the cardinality is $\leq e^{\varepsilon n} e^{4 \varepsilon P}$, and from the proof of [8, Sublemma 2.9] $n_{i+1}-n_{i} \leq 2 p_{i}$ holds for every $1 \leq i \leq s$, where $n_{s+1}>k+l$ is such that $\tilde{\mathcal{P}}_{n_{s+1}}$ partitions $\omega_{n_{s}}$. It follows that $l<n_{s+1} \leq n_{1}+2 \sum_{i=1}^{s} p_{i}$. If $n_{1} \leq k+l / 2$ then $\sum_{i=1}^{s} p_{i} \geq l / 4$, and therefore

$$
\sum_{\substack{\eta \in \mathcal{Q}^{\prime} \\ n_{1} \leq k+l / 2}}|\eta| \leq C_{0} \delta^{-1} \frac{l}{N} \sum_{P \geq l / 4} e^{\left(8 \varepsilon-\frac{\lambda}{3}\right) P} \frac{|\omega|}{\left|f^{k} \omega\right|} \leq e^{-\frac{\lambda}{13} l} \frac{|\omega|}{\left|f^{k} \omega\right|}
$$

where the last inequality holds provided $k$ is sufficiently large because $l \geq \sqrt{\varepsilon} k$.

For those $\eta \in \mathcal{Q}^{\prime}$ with $n_{1}>k+l / 2$, a similar reasoning shows

$$
|\eta| \leq\left|\omega_{n_{1}}\right| \leq C_{0} \delta^{-1} e^{-\lambda\left(n_{1}-k\right)} \frac{|\omega|}{\left|f^{k} \omega\right|} \leq C_{0} \delta^{-1} e^{-\frac{\lambda l}{2}} \frac{|\omega|}{\left|f^{k} \omega\right|} \leq e^{-\frac{\lambda l}{3}} \frac{|\omega|}{\left|f^{k} \omega\right|}
$$

and therefore

$$
\sum_{\substack{\eta \in \mathcal{Q}^{\prime} \\ n_{1}>k+l / 2}}|\eta| \leq C_{0} \delta^{-1} \frac{l}{N} \sum_{P \leq l} e^{4 \varepsilon P+\varepsilon l-\frac{\lambda}{3} l} \frac{|\omega|}{\left|f^{k} \omega\right|} \leq e^{-\frac{\lambda l}{4}} \frac{|\omega|}{\left|f^{k} \omega\right|} .
$$

We now treat elements of $\mathcal{Q}^{\prime \prime}$. Let $t_{1} \geq k$ be such that $\omega$ is subdivided at time $t_{1}$. Since $I_{p, j} \supset f^{n} \omega$ holds for some $n<k$ we have $\left|f^{k} \omega\right| \geq \delta e^{-5 \varepsilon k}$. If $t_{1}-k \geq 16(\varepsilon / \lambda) k$, then $\left|f^{t_{1}} \omega\right| \geq \delta e^{-5 \varepsilon k} e^{\frac{\lambda}{3}\left(t_{1}-k\right)} \geq \delta e^{\varepsilon k}>2=|X|$, which is a contradiction. Hence $t_{1}-k<(16 \varepsilon / \lambda) k$, and so $t_{1}<k+l$.

Let $t \geq k$. We say $\tilde{\omega} \in \tilde{\mathcal{P}}_{t} \mid \omega$ is an escaping component at time $t$ if $\tilde{\omega}$ arises out of subdivision at time $t$ and satisfies $d\left(0, f^{t} \tilde{\omega}\right)=\delta$. Let $\mathcal{E}_{1}$ denote the collection of escaping components at time $k+r$. If $\mathcal{E}_{1}=\emptyset$, then $\mathcal{Q}^{\prime \prime}=\emptyset$. Hence we assume $\mathcal{E}_{1} \neq \emptyset$.

Each $\eta \in \mathcal{Q}^{\prime \prime}$ has an itinerary $\left(t_{1}, \epsilon_{1}\right), \ldots,\left(t_{q}, \epsilon_{q}\right)$ that is defined as follows: $k \leq t_{1}<\cdots<$ $t_{q}<k+l$ is a sequence of integers, associated with a nested sequence $\omega \supset \omega_{t_{1}} \supset \cdots \supset \omega_{t_{q}} \supset \eta$ of intervals such that for each $i, \omega_{t_{i}}$ is an escaping component at time $t_{i}$ and $\epsilon_{i}=+$ (resp. $\left.\varepsilon_{i}=-\right)$ if $f^{t_{i}} \omega_{t_{i}}$ is at the right (resp. left) of the critical point; $\omega_{t_{q}}$ is the smallest escaping component containing $\eta$. Call $q$ the length of the itinerary of $\eta$.

For $\theta>0$ let $\mathcal{Q}_{\leq \theta l}^{\prime \prime}=\left\{\eta \in \mathcal{Q}^{\prime \prime}\right.$ : The length of the itinerary is $\left.\leq \theta l\right\}$. The number of all itineraries of length $q$ is $\leq 2^{q}\left(\begin{array}{l}l \\ q\end{array}\right)$, and so the Stirling formula implies one can choose small $\theta$ such that $\# \mathcal{Q}_{\leq \theta l}^{\prime \prime} \leq e^{\lambda l / 100}$. Then

$$
\sum_{\eta \in \mathcal{Q}_{\leq \theta l}^{\prime \prime}}|\eta| \leq \# \mathcal{Q}_{\theta l}^{\prime \prime} e^{-\lambda l / 2} \frac{|\omega|}{\left|f^{k} \omega\right|} \leq e^{-\frac{\lambda}{3} l} \frac{|\omega|}{\left|f^{k} \omega\right|}
$$

To treat elements in $\mathcal{Q}_{>\theta l}^{\prime \prime}=\left\{\eta \in \mathcal{Q}^{\prime \prime}\right.$ : The length of the itinerary is $\left.>\theta l\right\}$, for each $q \geq 1$ define a collection $\mathcal{E}_{q}$ of escaping components (at variable times) inductively as follows: each $\omega \in \mathcal{E}_{q}$ is an escaping component at some time, say $t=t(\omega)$. Let $t^{\prime}>t$ denote the time at which $\omega$ is subdivided. Then $\omega$ contains no or at most two escaping components at time $t^{\prime}$. 
We let them in $\mathcal{E}_{q+1}$. Let $E_{q}=\bigcup_{\omega \in \mathcal{E}_{q}} \omega$. If $\omega \in \mathcal{E}_{q}$ and $\omega \cap E_{q+1} \neq \emptyset$, then from the bounded distortion (6),

$$
\frac{\left|\omega \backslash E_{q+1}\right|}{|\omega|} \geq C_{0}^{-1} \frac{\left|f^{t^{\prime}}\left(\omega \backslash E_{q+1}\right)\right|}{\left|f^{t^{\prime}} \omega\right|} \geq C_{0}^{-1} \frac{\left|\Lambda^{+}\right|}{|X|}=\hat{\zeta}
$$

and so $\left|\omega \cap E_{q+1}\right| \leq|\omega|-\left|\omega \backslash E_{q+1}\right| \leq(1-\hat{\zeta})|\omega|$. Hence $\left|E_{q+1}\right| \leq(1-\hat{\zeta})\left|E_{q}\right|$, and thus $\left|E_{q}\right| \leq(1-\hat{\zeta})^{q}|\omega|$. By definition, if the itinerary of $\eta \in \mathcal{Q}_{>\theta l}^{\prime \prime}$ is of length $q$, then $\eta$ is contained in an element of $\mathcal{E}_{q}$. Hence

$$
\sum_{\eta \in \mathcal{Q}_{>\theta l}^{\prime \prime}}|\eta| \leq \sum_{\theta l \leq q \leq l}\left|E_{q}\right| \leq \sum_{q \geq \theta l}(1-\hat{\zeta})^{q} \leq \hat{\zeta}^{-1}(1-\hat{\zeta})^{\theta l}
$$

Since $\theta$ is independent of $\delta$ and $\hat{\zeta} \rightarrow 0$ as $\delta \rightarrow 0$, we have $e^{-\frac{\lambda}{14}} \leq \zeta$. (7) (18) (19) (10) yield $|\{R>k+l\} \cap \omega| \leq e^{-\frac{\lambda}{14} l}+\hat{\zeta}^{-1}(1-\hat{\zeta})^{\theta l} \leq C_{1} \zeta^{l}$.

2.5. Bounded distortion. We prove a statement on distortions. Let $J$ be an interval. A differentiable map $g: J \rightarrow \mathbb{R}$ without a critical point has distortion bounded by $\kappa \geq 1$ if

$$
\sup _{x, y \in J} \frac{|D g(x)|}{|D g(y)|} \leq \kappa \text {. }
$$

Let $J \subset T$ be two intervals and $n>0$ such that $f^{n} \mid T$ is strictly monotone. We say $f^{n} T$ contains a $\xi$-scaled neighborhood of $f^{n} J$ if the lengths of both components of $f^{n}(T \backslash J)$ are $\geq \xi\left|f^{n} J\right|$. The following is known as the Koebe Principle [23, Chapter IV.1].

Lemma 2.5. Let $J \subset T$ be two intervals and $n>0$ such that $f^{n} \mid T$ is strictly monotone and $f^{n} T$ contains a $\xi$-scaled neighborhood of $f^{n} J$. Then $f^{n} \mid J$ has distortion bounded by $((1+\xi) / \xi)^{2}$.

Let $J \subset \hat{X}$ be an interval and $n>0$. We say $f^{n} J$ is a free segment (resp. bound segment) if it is the union of elements of $\tilde{\mathcal{P}}_{n}$, and for any $\omega \in \tilde{\mathcal{P}}_{n} \mid J, f^{n} \omega$ is free (resp. bound). A free segment $f^{n} J$ is maximal if it there is no interval $I \subset \hat{X}$ containing $J$ such that $f^{n} I$ is a free segment.

Lemma 2.6. If $n \geq N$ and $f^{n} J$ is a maximal free segment not containing $\{\hat{x},-\hat{x}\}$, then there is an interval $T \supset J$ such that $f^{n} \mid T$ is strictly monotone and $f^{n} T$ contains a $e^{-6 \varepsilon n}$-scaled neighborhood of $f^{n} J$. In particular, $f^{n} \mid J$ has distortion bounded by $e^{13 \varepsilon n}$.

Proof. By the assumption, to each side of $J$ is attached an interval $\omega \in \tilde{\mathcal{P}}_{n}$ such that $f^{n} \omega$ is bound. Let $k$ denote the maximal $i<n$ such that $f^{i} \omega$ is free and set $p=p_{i}(\omega)$. Then $k<n<k+p$. If $k+p=n+1$, then using $|D f| \leq 4$ and [ㅇ, Lemma 2.6(a)] we have $\left|f^{n} \omega\right| \geq(1 / 4)\left|f^{n+1} \omega\right|=(1 / 4)\left|f^{k+p} \omega\right| \geq(1 / 4) e^{-5 \varepsilon p} \geq e^{-6 \varepsilon n}$. If $k+p>n+1$, then $p=N$ and so $\left|f^{n} \omega\right| \geq(1 / 4)^{k+p-n}\left|f^{k+p} \omega\right| \geq(1 / 4)^{N}\left|f^{k+p} \omega\right| \geq(1 / 4)^{N} e^{-5 \varepsilon N} \geq e^{-6 \varepsilon n}$.

2.6. Construction of finite partitions. The partition $\tilde{\mathcal{P}}_{n}$ restricted to $\{R>n\}$ is actually too fine to be used for an upper estimate of the Hausdorff dimension. Hence we construct a finite partition $\mathcal{P}_{n}$ by gluing some elements of $\tilde{\mathcal{P}}_{n}$.

Start with $\mathcal{P}_{0}=\left\{\Lambda^{-}, \Lambda^{+}\right\}$. Assume inductively that $\mathcal{P}_{n-1}$ has been constructed with the following properties:

$(\mathrm{P} 1)_{n-1}$ it is a partition of the set $\{R>n-1\}$ into a finite number of intervals each of which is the union of a countable number of elements of $\tilde{\mathcal{P}}_{n-1} \mid\{R>n-1\}$; 
$(\mathrm{P} 2)_{n-1}$ for any $\omega \in \mathcal{P}_{n-1}$ let

$$
\operatorname{fr}(\omega)=\bigcup\left\{\omega^{\prime} \in \tilde{\mathcal{P}}_{n-1} \mid \omega: f^{n} \omega^{\prime} \text { is free }\right\} \text { and } \operatorname{bo}(\omega)=\bigcup\left\{\omega^{\prime} \in \tilde{\mathcal{P}}_{n-1} \mid \omega: f^{n} \omega^{\prime} \text { is bound }\right\} .
$$

These two sets are intervals unless empty. In addition, $\operatorname{fr}(\omega)$ is the union of at most $e^{3 \varepsilon(n-1)}$ number of elements of $\tilde{\mathcal{P}}_{n-1}$.

Let $\omega \in \mathcal{P}_{n-1}$ and write $\{R=n\}=\{R>n-1\} \backslash\{R>n\}$. The partition $\mathcal{P}_{n}$ on $\omega \backslash\{R=n\}$ is defined as follows. Let $\mathrm{bo}(\omega) \in \mathcal{P}_{n}$ unless empty. If $\operatorname{fr}(\omega) \neq \emptyset$ then there are two cases:

- if $\operatorname{fr}(\omega) \cap\{R=n\}=\emptyset$, then define $\mathcal{P}_{n} \mid \operatorname{fr}(\omega)$ by dividing $f^{n} \operatorname{fr}(\omega)$ into at most two intervals, one which is at the right of 0 and the other at the left of 0 ;

- if $\operatorname{fr}(\omega) \cap\{R=n\} \neq \emptyset$, then $\operatorname{fr}(\omega) \backslash\{R=n\}$ consists of at most three intervals $\omega^{-}$, $\omega^{+}, \omega^{0}$, where the corresponding $f^{n}$-images are: at the left of $\Lambda^{-}$; at the right of $\Lambda^{+}$; in between $\Lambda^{-}$and $\Lambda^{+}$. Let $\omega^{ \pm} \in \mathcal{P}_{n}$ unless empty. Finally define $\mathcal{P}_{n} \mid \omega^{0}$ by dividing $f^{n} \omega^{0}$ into at most two intervals, one which is at the right of 0 and the other at the left of 0 .

This completes the definition of $\mathcal{P}_{n}$. $(\mathrm{P} 1)_{n}$ holds by construction. To see $(\mathrm{P} 2)_{n}$, let $\omega \in \mathcal{P}_{n}$. The subdivision algorithm described in Sect.2.3 and the "monotonicity" of the bound periods with respect to the distance to the critical point imply that $\operatorname{fr}(\omega), \mathrm{bo}(\omega)$ are intervals or empty sets. By construction, $\operatorname{fr}(\omega) \in \tilde{\mathcal{P}}_{n}$, or else it is made up of elements of $\tilde{\mathcal{P}}_{n}$ with the same latest bound period at the same time $k, k<n+1$. Hence $\operatorname{fr}(\omega)$ is the union of at most $e^{3 \varepsilon n}$ elements of $\tilde{\mathcal{P}}_{n}$.

2.7. Abundance of long free segments. The next lemma allows us to find long free segments in generic partition elements.

Lemma 2.7. There exists $k_{1}=k_{1}(\delta)>0$ such that if $k \geq k_{1}$ and $\omega \in \mathcal{P}_{k}$, then there exist $q \in[k+1,(1+3 \varepsilon / \lambda) k]$ and $\omega^{\prime} \in \tilde{\mathcal{P}}_{q} \mid \omega$ such that:

(a) $f^{q} \omega^{\prime}$ is free;

(b) $\left|\omega^{\prime}\right| \geq e^{-18 \varepsilon k}|\omega|$;

(c) $\omega^{\prime} \subset\{R>q-1\}$.

Proof. We first consider the case $|\operatorname{fr}(\omega)| \geq(1 / 2)|\omega|$. By $(\mathrm{P} 2)_{k}, \operatorname{fr}(\omega)$ is the union of at most $e^{3 \varepsilon k}$ number of elements of $\tilde{\mathcal{P}}_{k}$. Hence it is possible to choose $\omega^{\prime} \in \tilde{\mathcal{P}}_{k} \mid \operatorname{fr}(\omega)$ such that $\left|\omega^{\prime}\right| \geq$ $(1 / 2) e^{-3 \varepsilon k}|\omega|$. Set $q=k+1$. Then (a) (b) hold. (c) is because $\omega \subset\{R>k\}=\{R>q-1\}$.

We now consider the case $|\mathrm{bo}(\omega)| \geq(1 / 2)|\omega|$. In this case we shall choose $\omega^{\prime}$ to be a certain subinterval of bo $(\omega)$. Let $i$ denote the maximal $j \leq k$ such that $f^{j} \operatorname{bo}(\omega)$ is a free segment. Let $r$ denote the minimum of the bound period $p_{i}: \tilde{\mathcal{P}}_{i} \mid \operatorname{bo}(\omega) \rightarrow \mathbb{N}$ at time $i$. Set $q=i+r$.

Sublemma 2.8. $r \leq k$ and $k+1<q \leq(1+3 / \lambda) k$.

Proof. Since $f^{k+1} \mathrm{bo}(\omega)$ is a bound segment, $k+1<q$. To show the rest, for $i-1 \leq j \leq k+1$ let $\omega_{j}$ denote the element of $\mathcal{P}_{j}$ containing bo $(\omega)$. We have $\omega_{i-1} \supset \omega_{i} \supset \cdots \supset \omega_{k} \supset \omega_{k+1}$, $\omega_{k}=\omega$ and $\omega_{k+1}=\operatorname{bo}(\omega)$. Note that $\tilde{\mathcal{P}}_{j} \mid \omega_{j}=\left\{\omega^{\prime} \in \tilde{\mathcal{P}}_{i} \mid \omega_{i}: p_{i}\left(\omega^{\prime}\right)>j-i\right\}$. We treat two cases separately.

Case $I: \omega_{i}=\operatorname{bo}(\omega)$. Since $f^{i} \operatorname{bo}(\omega)$ is a free segment, $\omega_{i} \subset \operatorname{fr}\left(\omega_{i-1}\right)$. Since $\operatorname{fr}\left(\omega_{i-1}\right)$ is the union of elements of $\tilde{\mathcal{P}}_{i-1}$, for any point $x$ in the boundary of $\operatorname{fr}\left(\omega_{i-1}\right)$ we have $\left|f^{i} x\right| \geq \delta e^{-\varepsilon i}$. In other words, $f^{i} f r\left(\omega_{i-1}\right)$ is not contained in $\left(-\delta e^{-\varepsilon i}, \delta e^{-\varepsilon i}\right)$, and the same holds for $f^{i} \omega_{i}$. By 
Lemma 2.2(b) we have $r \leq(3 \varepsilon / \lambda) i<k$, and so $q \leq k+r \leq(1+3 \varepsilon / \lambda) k$ for sufficiently large $k$.

Case II: $\omega_{i} \supsetneq \operatorname{bo}(\omega)$. Let $r^{\prime}$ denote the mimimum of the bound period $p_{i}: \tilde{\mathcal{P}}_{i} \mid \omega_{i} \rightarrow \mathbb{N}$ at time $i$. If $i+r^{\prime} \leq k+1$, then the monotonicity of the bound period implies $\omega_{i}=\cdots=\omega_{i+r^{\prime}-1} \supsetneq$ $\omega_{i+r^{\prime}} \supsetneq \cdots \supsetneq \omega_{k+1}$, and $\omega_{k+1} \notin \mathcal{P}_{k+2}$. This implies $r=k+2-i$, and so $q=k+2 \leq(1+3 \varepsilon / \lambda) k$. If $i+r^{\prime}>k+1$, then $\operatorname{fr}(\omega) \cap \omega_{i}=\emptyset$, and thus all $\operatorname{fr}(\omega)$, bo $(\omega), \omega_{i}$ share exactly one boundary point. Since $f^{i}$ sends $\omega$ diffeomorphically onto its image, $f^{i} \mathrm{bo}(\omega)$ must come close to the boundary of $(-\delta, \delta)$ so that $r=N$. Hence $q \leq(1+3 \varepsilon / \lambda) k$.

Choose $\omega^{\prime} \in \tilde{\mathcal{P}}_{i} \mid \operatorname{bo}(\omega)$ such that $r=p_{i}\left(\omega^{\prime}\right)$. Then $f^{q} \omega^{\prime}$ is free, and $\omega^{\prime} \subset\{R>q-1\}$. As for (b), since $f^{i} \omega^{\prime}$ contains some $I_{r, j}$ we have $\left|f^{i} \omega^{\prime}\right| \geq\left(\delta_{r-1}-\delta_{r}\right) e^{-3 \varepsilon r}$. Since $\left|f^{i} \mathrm{bo}(\omega)\right| \leq \delta_{r-1}$ and $\delta_{r} \leq e^{-\frac{\varepsilon}{2}} \delta_{r-1}$,

$$
\frac{\left|f^{i} \omega^{\prime}\right|}{\left|f^{i} \mathrm{bo}(\omega)\right|} \geq \frac{\left(\delta_{r-1}-\delta_{r}\right) e^{-3 \varepsilon r}}{\delta_{r-1}} \geq\left(1-e^{-\frac{\varepsilon}{2}}\right) e^{-3 \varepsilon r} .
$$

Suppose that bo $(\omega)$ is contained in an interval which does not contain $\{ \pm \hat{x}\}$ and whose $f^{i}$ image is a maximal free segment. By Lemma 2.6 and $r \leq k$ in Sublemma 2.8,

$$
\left|\omega^{\prime}\right| \geq e^{-13 \varepsilon i}\left(1-e^{-\frac{\varepsilon}{2}}\right) e^{-3 \varepsilon r}|\operatorname{bo}(\omega)| \geq e^{-17 \varepsilon k}|\operatorname{bo}(\omega)| \geq(1 / 2) e^{-17 \varepsilon k}|\omega| \geq e^{-18 \varepsilon k}|\omega| .
$$

Even if the above is not the case, the proof of Lemma 2.6 implies essentially the same distortion bounds, and so the same lower estimate of $\left|\omega^{\prime}\right|$ holds.

2.8. Special property of the partition. The next lemma asserts that a positive definite fraction of points in each element of $\mathcal{P}_{k}$ quickly return to the base $\Lambda$.

Lemma 2.9. There exists $k_{2} \geq \max \left\{k_{0}, k_{1}\right\}$ such that if $k \geq k_{2}$ and $\omega \in \mathcal{P}_{k}$, then there exists $\tilde{\omega} \in \mathcal{Q}$ such that:

(a) $\tilde{\omega} \subset \omega$ and $|\tilde{\omega}| \geq e^{-\sqrt{\varepsilon} k}|\omega|$;

(b) $k<R(\tilde{\omega}) \leq(1+19 \varepsilon / \lambda) k$.

Proof. Choose $q \in[k+1,(1+3 \varepsilon / \lambda) k]$ and $\omega^{\prime} \in \tilde{\mathcal{P}}_{q} \mid \omega$ for which the conclusions of Lemma 2.7 holds. By Lemma 2.4,

$$
\left|\omega^{\prime} \cap\{R<q+(16 \varepsilon / \lambda) k\}\right| \geq\left(1-C_{1} \zeta^{k}\right)\left|\omega^{\prime}\right| \geq(1 / 2)\left|\omega^{\prime}\right| .
$$

Since the $f^{r}$-image of $f^{q} \omega^{\prime}$ is folded at most $2^{r}$ times, $\#\left\{\omega \in \mathcal{Q} \mid \omega^{\prime}: R(\omega)=q+r\right\} \leq 2^{r+1}$ and SO

$$
\begin{aligned}
\#\left\{\omega \in \mathcal{Q} \mid \omega^{\prime}: R(\omega)<q+(16 \varepsilon / \lambda) k\right\} & =\sum_{r=0}^{[(16 \varepsilon / \lambda) k]} \#\left\{\omega \in \mathcal{Q} \mid \omega^{\prime}: R(\omega)=q+r\right\} \\
& \leq \sum_{r=0}^{[(16 \varepsilon / \lambda) k]} 2^{r+1} \leq e^{(17 \varepsilon / \lambda) k},
\end{aligned}
$$

where the last inequality holds for sufficiently large $k$. Then it is possible to choose $r \leq$ $(16 \varepsilon / \lambda) k$ and $\tilde{\omega} \in \mathcal{Q} \mid \omega^{\prime}$ such that $R(\tilde{\omega})=q+r \leq(1+19 \varepsilon / \lambda) k$ and $|\tilde{\omega}| \geq(1 / 2) e^{-(17 \varepsilon / \lambda) k}\left|\omega^{\prime}\right|$. From this and Lemma 2.7(b) we obtain (a). 
2.9. Towers. We now translate Lemma 2.9 into the language of towers. By Lemma 2.4, we may think of $R: \mathcal{Q} \rightarrow \mathbb{N}$ as a function on a full measure subset of $\Lambda$ in the obvious way. Let

$$
\Delta=\{(x, \ell): x \in \Lambda, \quad \ell=0,1, \ldots, R(x)-1\},
$$

which we call a tower, and define a tower map $\hat{f}: \Delta \circlearrowleft$ by

$$
\hat{f}(x, \ell)= \begin{cases}(x, \ell+1) & \text { if } \ell+1<R(x) \\ \left(f^{R(x)} x, 0\right) & \text { if } \ell+1=R(x) .\end{cases}
$$

The point $(x, \ell)$ is considered to be climbing the tower in the first case, and falling down from the tower in the second case. Define $\Delta_{\ell}=\{(x, \ell) \in \Delta: R(x)>\ell\}$. With the canonical identification $\{R>\ell\} \ni x \mapsto(x, \ell) \in \Delta_{\ell}$ we transplant the partition $\mathcal{P}_{\ell}$ of $\{R>\ell\}$ to the partition of $\Delta_{\ell}$ and also denote it by $\mathcal{P}_{\ell}$. Let $\mathcal{D}=\bigcup_{\ell>0} \mathcal{P}_{\ell}$. This is a partition of $\Delta$ with a Markov property: for any $\omega \in \mathcal{D}, \hat{f} \omega$ is a finite union of elements of $\mathcal{D}$. We identify $\Delta_{0}=\{(x, 0): x \in \Lambda\}$ with $\Lambda$ under the action of the map $\pi: \Delta \rightarrow \Lambda$ given by $\pi(x, \ell)=x$.

Lemma 2.10. The following holds for sufficiently large $n$ : for any $A \in \bigvee_{i=0}^{n-1} \hat{f}^{-i} \mathcal{D}$ with $A \subset \Delta_{0}$ there exist an interval $\tilde{A} \subset \Delta_{0}$ and $t \in[(1-\varepsilon) n,(1+20 \varepsilon / \lambda) n]$ such that:

(a) $\tilde{A} \subset A$, and $\hat{f}^{t} \tilde{A}=\Lambda^{+}$or $=\Lambda^{-}$;

(b) $|\tilde{A}| \geq e^{-2 \sqrt{\varepsilon} n}|A|$.

Proof. In the first $n$-iterates under $\hat{f}$, the interval $A$ continues climbing the tower, or else falls down from the tower several times. Let $j=\max \left\{i \geq 0: \hat{f}^{i} A \subset \Delta_{0}\right\}$. Since $f^{j} A \subset \Lambda^{ \pm}$ and $f^{j} \mid A$ is extended to a diffeomorphism onto $3 \Lambda^{ \pm}, f^{j} \mid A$ has distortion bounded by 4 . Set $\omega=f^{j} A$ and $k=n-j-1$. Since $A \subset \Delta_{0}$ we have $\omega \in \mathcal{P}_{k}$. If $k \geq k_{2}$, then take a subinterval $\tilde{\omega} \subset \omega$ for which the conclusions of Lemma 2.9 hold, and define $t=j+R(\tilde{\omega})$. The bounds on $t$ follow from Lemma 2.9(b). Define $\tilde{A}$ to be the subinterval of $A$ be such that $\tilde{\omega}=f^{j} \tilde{A}$. Then

$$
\frac{|\tilde{A}|}{|A|} \geq \frac{1}{4} \frac{|\tilde{\omega}|}{|\omega|} \geq \frac{1}{4} e^{-\sqrt{\varepsilon} k} \geq e^{-2 \sqrt{\varepsilon} n} .
$$

The second inequality follows from Lemma 2.9(a). If $k<k_{2}$, then set $t=j$ and define $\tilde{A}$ to be the subinterval of $A$ such that $f^{t} \tilde{A}=\Lambda^{ \pm}$.

\section{UPPER ESTIMATE OF BIRKhofF SPECTRUM}

We put together the constructions and the results in Sect.2 to obtain an upper estimate of the Birkhoff spectrum. For $\varphi \in C(X), k \geq 0, \alpha \in\left[c_{\varphi}, d_{\varphi}\right]$ and $\varepsilon>0$ consider the set

$$
\Gamma_{k}=\Gamma_{k}(\varphi ; \alpha, \varepsilon)=\left\{x \in \Lambda:\left|\frac{1}{n} S_{n} \varphi(x)-\alpha\right|<\varepsilon \text { for every } n \geq k\right\} .
$$

Note that $\Gamma_{k}$ is increasing in $k$. Since $K_{\varphi}(\alpha)$ is dense in $X$ and $\Lambda$ contains open sets, $\Gamma_{k} \neq \emptyset$ holds for sufficiently large $k$. Define $\sigma=\sigma(\varphi ; \alpha, \varepsilon)$ by

$$
\sigma=\sup \left\{\frac{h(\mu)}{\lambda(\mu)}: \mu \in \mathcal{M}_{f},|\mu(\varphi)-\alpha| \leq \sqrt{\varepsilon}\right\}+\varepsilon^{\frac{1}{3}} .
$$

Since $\lambda_{\text {inf }}>0, \sigma$ stays bounded from above as $\varepsilon \rightarrow 0$. 
Proposition 3.1. If $\varphi \in C(X)$ is Lipschitz, then for any $\alpha \in\left[c_{\varphi}, d_{\varphi}\right]$ and $\varepsilon>0$,

$$
\operatorname{dim}_{H} \Gamma_{k}(\varphi ; \alpha, \varepsilon) \leq \sigma(\varphi ; \alpha, \varepsilon) \quad \text { for every } k \geq 0
$$

We finish the upper estimate of $B_{\varphi}(\alpha)$ assuming the conclusion of Proposition 3.1. Set $Y=\left[f^{2} 0, f 0\right]$. Points in $X \backslash Y$ are mapped to $Y$ by some positive iterates. The countable stability and the invariance of Hausdorff dimension under the action of Lipschitz continuous homeomorphisms yields $B_{\varphi}(\alpha)=\operatorname{dim}_{H}\left(K_{\varphi}(\alpha) \cap Y\right)$. We estimate the right-hand-side.

By (A4) there exists $M>0$ such that $f^{M} \Lambda=Y$. Then

$$
\operatorname{dim}_{H}\left(K_{\varphi}(\alpha) \cap Y\right)=\operatorname{dim}_{H} f^{M}\left(K_{\varphi}(\alpha) \cap \Lambda\right) \leq \operatorname{dim}_{H}\left(K_{\varphi}(\alpha) \cap \Lambda\right) \leq \lim _{k \rightarrow \infty} \operatorname{dim}_{H} \Gamma_{k},
$$

where the last inequality is because $K_{\varphi}(\alpha) \cap \Lambda \subset \bigcup_{k \geq n} \Gamma_{k}$ for every $n \geq 0$. If $\varphi$ is Lipschitz continuous, then by Proposition 3.1.

$$
B_{\varphi}(\alpha) \leq \lim _{k \rightarrow \infty} \operatorname{dim}_{H} \Gamma_{k} \leq \sigma
$$

Letting $\varepsilon \rightarrow 0$ we get

$$
B_{\varphi}(\alpha) \leq \lim _{\varepsilon \rightarrow 0} \sup \left\{\frac{h(\mu)}{\lambda(\mu)}: \mu \in \mathcal{M}_{f},|\mu(\varphi)-\alpha|<\varepsilon\right\} .
$$

If $\varphi$ is merely continuous, then take a Lipschitz continuous $\tilde{\varphi}$ such that $\|\varphi-\tilde{\varphi}\|<\varepsilon / 2$, $c_{\tilde{\varphi}}=c_{\varphi}$ and $d_{\tilde{\varphi}}=d_{\varphi}$. Then for any $\alpha \in\left[c_{\varphi}, d_{\varphi}\right]$ and small $\varepsilon>0, \Gamma_{k}(\varphi ; \alpha, \varepsilon) \subset \Gamma_{k}(\tilde{\varphi} ; \alpha, 2 \varepsilon)$ holds. By Proposition 3.1 there exists $\xi \in \mathcal{M}_{f}$ such that

$$
\operatorname{dim}_{H} \Gamma_{k}(\varphi ; \alpha, \varepsilon) \leq \operatorname{dim}_{H} \Gamma_{k}(\tilde{\varphi} ; \alpha, 2 \varepsilon) \leq \frac{h(\xi)}{\lambda(\xi)}+(3 \varepsilon)^{\frac{1}{3}}
$$

and

$$
|\xi(\varphi)-\alpha| \leq|\xi(\varphi)-\xi(\tilde{\varphi})|+|\xi(\tilde{\varphi})-\alpha|<\varepsilon / 2+\sqrt{2 \varepsilon}<2 \sqrt{\varepsilon}
$$

The rest of the argument is identical to the previous case.

The rest of this section is entirely devoted to the proof of Proposition 3.1. In Sect 3.1 we extract from the towers uniformly hyperbolic invariant sets (horseshoes). In Sect 3.2 we construct invariant measures on the horseshoes, and use them to complete the proof of the proposition.

\subsection{Construction of a horseshoe. Define}

$$
\mathcal{A}_{n}=\left\{A \in \bigvee_{i=0}^{n-1} \hat{f}^{-i} \mathcal{D}: A \subset \Delta_{0},\left|\frac{1}{n} S_{n} \varphi(x)-\alpha\right|<\varepsilon \text { for some } x \in A\right\} \text {. }
$$

If $\Gamma_{k} \neq \emptyset$ then for every $n \geq k$ we have $\mathcal{A}_{n} \neq \emptyset$, and $\Gamma_{k} \subset \bigcup_{A \in \mathcal{A}_{n}} A$. We use this family of coverings for the upper estimate of the Hausdorff dimension.

Let $\Omega$ be a finite collection of pairwise disjoint closed intervals in $\Lambda$ and $r$ a positive integer. We say $\Omega$ generates a horseshoe for $f^{r}$ if $f^{r}$ sends each element of $\Omega$ diffeomorphically onto $\hat{X}$. By a horseshoe we mean the set

$$
H_{r}(\Omega)=\bigcap_{j=0}^{\infty}\left(f^{r}\right)^{-j}\left(\bigcup_{I \in \Omega} I\right)
$$


Lemma 3.2. For any $\varepsilon>0$ there exists $n^{\prime}>0$ such that if $n \geq n^{\prime}$ then $\mathcal{A}_{n} \neq \emptyset$ and there exist a finite collection $\mathcal{K}$ of closed intervals in $\Lambda$ and an integer $q \in[(1-\varepsilon) n,(1+21 \varepsilon / \lambda) n]$ such that:

(a) $\mathcal{K}$ generates a horseshoe for $f^{q}$;

(b) $\sum_{K \in \mathcal{K}}|K|^{\sigma} \geq e^{-3 \sqrt{\varepsilon} \sigma n} \sum_{A \in \mathcal{A}_{n}}|A|^{\sigma}$;

(c) for all $x \in H_{q}(\mathcal{K}),\left|(1 / q) S_{q} \varphi(x)-\alpha\right| \leq \sqrt{\varepsilon}$.

Proof. For each $A \in \mathcal{A}_{n}$, fix once and for all an interval $\tilde{A}$ and an integer $t=t_{A}$ for which the conclusions of Lemma 2.10 hold. Let $\mathcal{A}_{n}(t)=\left\{A \in \mathcal{A}_{n}: t_{A}=t\right\}$. Then $t_{A} \in[(1-\varepsilon) n,(1+$ $19 \varepsilon / \lambda) n]$. Let $t_{0}$ be a value of $t$ which maximizes $\sum_{A \in \mathcal{A}_{n}(t)}|\tilde{A}|^{\sigma}$. Then

$$
\sum_{A \in \mathcal{A}_{n}\left(t_{0}\right)}|\tilde{A}|^{\sigma} \geq \frac{1}{(1+20 / \lambda) \varepsilon n} \sum_{A \in \mathcal{A}_{n}}|\tilde{A}|^{\sigma} .
$$

By (A4) it is possible to choose a constant $\tau>0$, an integer $u>0$ and a closed interval $I^{+} \subset$ $\Lambda^{+}$such that $\Lambda^{+}$contains the $\tau$-scaled neighborhood of $I^{+}$, and $f^{u}$ sends $I^{+}$diffeomorphically onto $\hat{X}$. Define $q=t_{0}+u$. The bounds on $q$ hold for sufficiently large $n$.

Let $I^{-}=-I^{+}$. For each $A \in \mathcal{A}_{n}\left(t_{0}\right)$ define $K(A)$ to be the preimage of $I^{+}$or $I^{-}$under $f^{t_{0}} \mid \tilde{A}$, according to whether $f^{t_{0}} \tilde{A}=\Lambda^{+}$or $=\Lambda^{-}$. Set $\mathcal{K}=\left\{K(A): A \in \mathcal{A}_{n}\left(t_{0}\right)\right\}$. Then $\mathcal{K}$ is a finite collection of pairwise disjoint closed intervals in $\Lambda$, and $f^{q}$ sends each element of $\mathcal{K}$ diffeomorphically onto $\hat{X}$. Set $c=(\tau /(1+\tau))^{2}\left|I^{+}\right| /\left|\Lambda^{+}\right|$. Then

$$
\begin{aligned}
\sum_{K \in \mathcal{K}}|K|^{\sigma} & \geq c^{\sigma} \sum_{A \in \mathcal{A}_{n}\left(t_{0}\right)}|\tilde{A}|^{\sigma} \geq \frac{c^{\sigma}}{(1+20 / \lambda) \varepsilon n} \sum_{A \in \mathcal{A}_{n}}|\tilde{A}|^{\sigma} \\
& \geq \frac{1}{(1+20 / \lambda) \varepsilon n}\left(c e^{-2 \sqrt{\varepsilon} n}\right)^{\sigma} \sum_{A \in \mathcal{A}_{n}}|A|^{\sigma} \geq e^{-3 \sqrt{\varepsilon} \sigma n} \sum_{A \in \mathcal{A}_{n}}|A|^{\sigma} .
\end{aligned}
$$

Sublemma 3.3. There exists a constant $C>0$ such that if $n \geq N$ and $\omega \in \mathcal{P}_{n-1}$, then for all $x, y \in \omega$,

$$
\left|S_{n} \varphi(x)-S_{n} \varphi(y)\right| \leq \operatorname{Lip}(\varphi) \cdot C \delta^{-1},
$$

where $\operatorname{Lip}(\varphi)$ denotes the Lipschitz constant of $\varphi$.

Proof. Let $0 \leq i \leq n-1$. We call $f^{i} \omega$ free if there exists an interval $J \subset \hat{X}$ containing $\omega$ such that $f^{i} J$ is a free segment. Let $i_{0}$ denote the maximal $i \leq n-1$ such that $f^{i} \omega$ is free. From the construction in Sect.2 one can find integers $0 \leq r_{1}<\cdots<r_{s}=i_{0}, p_{1}, \ldots, p_{s}$ such that: $r_{1}$ is the smallest $i \geq 0$ with $f^{i} \omega \cap(-\delta, \delta) \neq \emptyset ; \delta_{p_{k}} \leq d\left(0, f^{r_{k}} \omega\right) \leq \delta_{p_{k}-2}$ and $r_{k+1}$ is the smallest $i \geq r_{k}+p_{k}$ with $f^{i} \omega \cap(-\delta, \delta) \neq \emptyset(k=1, \ldots, s-1) ; d\left(0, f^{r_{s}} \omega\right) \leq \delta_{p_{s}-2}$ and $n \leq r_{s}+p_{s}$. Then, similarly to the proof of [8, Sublemma 3.16] one can show that

$$
\sum_{i=0}^{n-1}\left|f^{i} \omega\right| \leq C \delta^{-1}
$$

This implies the desired inequality since $\varphi$ is Lipschitz continuous.

To prove (c), for each $A \in \mathcal{A}_{n}\left(t_{0}\right)$ pick $x_{A} \in A$ such that $\left|(1 / n) S_{n} \varphi\left(x_{A}\right)-\alpha\right| \leq \varepsilon$. We have

$$
S_{q} \varphi\left(x_{A}\right) \geq S_{n} \varphi\left(x_{A}\right)-\sup |\varphi| \cdot|q-n| \geq \alpha n-\sup |\varphi| \cdot(21 \varepsilon / \lambda) n \geq(\alpha-\sqrt{\varepsilon} / 2) q .
$$


In the same way we have $S_{q} \varphi\left(x_{A}\right) \leq(\alpha+\sqrt{\varepsilon} / 2) q$. Then

$$
\left|S_{q} \varphi\left(x_{A}\right)-q \alpha\right| \leq \sqrt{\varepsilon} \cdot q / 2 \text {. }
$$

By Sublemma 3.3, for any $x \in A$ we have $\left|S_{t_{0}} \varphi\left(x_{A}\right)-S_{t_{0}} \varphi(x)\right| \leq \operatorname{Lip}(\varphi) \cdot C \delta^{-1}$. Since $q-t_{0}=u$ and $q \geq(1-\varepsilon) n$, for sufficiently large $n$ we have

$$
\left|S_{q} \varphi\left(x_{A}\right)-S_{q} \varphi(x)\right| \leq \operatorname{Lip}(\varphi) \cdot C \delta^{-1}+2 \sup |\varphi| \cdot\left(q-t_{0}\right) \leq \sqrt{\varepsilon} \cdot q / 2 .
$$

(14) (15) yield $\left|S_{q} \varphi(x)-q \alpha\right| \leq \sqrt{\varepsilon} \cdot q$.

3.2. Construction of a measure on the horseshoe. For sufficiently large $n$, choose a finite collection $\mathcal{K}$ of closed intervals in $\Lambda$ and a positive integer $q$ for which the conclusions of Lemma 3.2 hold. Set $F=f^{q}$. By construction, $F$ is uniformly expanding on each element of $\mathcal{K}$. Hence, $F \mid H_{q}(\mathcal{K})$ is topologically conjugate to the one-sided full shift on $\# \mathcal{K}$-symbols. Write $\mathcal{K}=\left\{K_{1}, \ldots, K_{\# \mathcal{K}}\right\}$. For $\ell>0$ and an $(\ell+1)$-string $\left(a_{0}, \ldots, a_{\ell}\right)$ of integers in $[1, \# \mathcal{K}]$, define an interval

$$
K_{a_{0} \cdots a_{\ell}}=K_{a_{0}} \cap F^{-1} K_{a_{1}} \cap \cdots \cap F^{-\ell} K_{a_{\ell}} .
$$

Set $\kappa=2 C_{0} \sup _{x, y \in I^{+}} \frac{\left|D f^{u}(x)\right|}{\left|D f^{u}(y)\right|}$. We have

$$
\frac{\left|K_{a_{0} \cdots a_{\ell}}\right|}{\left|K_{a_{0} \cdots a_{\ell-1}}\right|} \geq \frac{1}{2} \frac{\left|F^{\ell-1} K_{a_{0} \cdots a_{\ell}}\right|}{\left|F^{\ell-1} K_{a_{0} \cdots a_{\ell-1}}\right|}=\frac{1}{2} \frac{\left|\left\{x \in K_{a_{\ell-1}}: F x \in K_{a_{\ell}}\right\}\right|}{\left|K_{a_{\ell-1}}\right|} \geq \kappa^{-1}\left|K_{a_{\ell}}\right| .
$$

The first inequality follows from the Koebe Principle, and the second one from (66) and the definition of $I^{+}, u$. Then

$$
\begin{aligned}
\sum_{\left(a_{0}, \ldots, a_{\ell}\right)}\left|K_{a_{0} \cdots a_{\ell}}\right|^{\sigma} & =\sum_{\left(a_{0}, \ldots, a_{\ell-1}\right)}\left|K_{a_{0} \cdots a_{\ell-1}}\right|^{\sigma} \sum_{a_{\ell}} \frac{\left|K_{a_{0} \cdots a_{\ell}}\right|^{\sigma}}{\left|K_{a_{0} \cdots a_{\ell-1}}\right|^{\sigma}} \\
& \geq \kappa^{-\sigma} \sum_{i=1}^{\# \mathcal{K}}\left|K_{i}\right|^{\sigma} \sum_{\left(a_{0}, \cdots, a_{\ell-1}\right)}\left|K_{a_{0} \cdots a_{\ell-1}}\right|^{\sigma} \\
& \geq \cdots \geq\left(\kappa^{-\sigma} \sum_{i=1}^{\# \mathcal{K}}\left|K_{i}\right|^{\sigma}\right)^{\ell+1}
\end{aligned}
$$

This yields

$$
\varliminf_{\ell \rightarrow \infty} \frac{1}{\ell} \log \sum_{\left(a_{0}, \ldots, a_{\ell}\right)}\left|K_{a_{0} \cdots a_{\ell}}\right|^{\sigma} \geq \log \sum_{i=1}^{\# \mathcal{K}}\left|K_{i}\right|^{\sigma}-\sigma \log \kappa .
$$

Let $\nu_{a_{0} \cdots a_{\ell}}$ denote the uniform distribution on the orbit of the $(\ell+1)$-periodic point of $F$ in $K_{a_{0} \cdots a_{\ell}}$. Define an $F$-invariant probability measure $\nu_{\ell}$ supported on $H_{q}(\mathcal{K})$ by

$$
\nu_{\ell}=\rho_{\ell} \sum_{\left(a_{0}, \ldots, a_{\ell}\right)}\left|K_{a_{0} \cdots a_{\ell}}\right|^{\sigma} \nu_{a_{0} \cdots a_{\ell}}
$$

where $\rho_{\ell}=1 / \sum_{\left(a_{0}, \ldots, a_{\ell}\right)}\left|K_{a_{0} \cdots a_{\ell}}\right|^{\sigma}$ is the normalizing constant. Pick an accumulation point of the sequence $\left\{\nu_{\ell}\right\}$ and denote it by $\nu_{0}$. Taking a subsequence if necessary we may assume this convergence takes place for the entire sequence. Using the relation $\nu_{\ell}\left(K_{a_{0} \cdots a_{\ell}}\right)=\rho_{\ell}\left|K_{a_{0} \cdots a_{\ell}}\right|^{\sigma}$ 
and $\left|K_{a_{0} \cdots a_{\ell}}\right| \leq \kappa|\hat{X}| \exp \left\{-(\ell+1) \int \log |D F| d \nu_{a_{0} \cdots a_{\ell}}\right\}$ which follows from the bounded distortion we have

$$
\begin{aligned}
\log \sum_{\left(a_{0}, \ldots, a_{\ell}\right)}\left|K_{a_{0} \cdots a_{\ell}}\right|^{\sigma} & =\sum_{\left(a_{0}, \ldots, a_{\ell}\right)} \nu_{\ell}\left(K_{a_{0} \cdots a_{\ell}}\right)\left(-\log \nu_{\ell}\left(K_{a_{0} \cdots a_{\ell}}\right)+\sigma \log \left|K_{a_{0} \cdots a_{\ell}}\right|\right) \\
& \leq-\sum_{\left(a_{0}, \ldots, a_{\ell}\right)} \nu_{\ell}\left(K_{a_{0} \cdots a_{\ell}}\right) \log \nu_{\ell}\left(K_{a_{0} \cdots a_{\ell}}\right)-\sigma(\ell+1) \int \log |D F| d \nu_{\ell}+\sigma \log \kappa|\hat{X}|
\end{aligned}
$$

A slight modification of the argument in the proof of the Variational Principle [34, Theorem 9.10] shows that

$$
\varlimsup_{\ell \rightarrow \infty} \frac{1}{\ell} \log \sum_{\left(a_{0}, \ldots, a_{\ell}\right)}\left|K_{a_{0} \cdots a_{\ell}}\right|^{\sigma} \leq h_{F}\left(\nu_{0}\right)-\sigma \int \log |D F| d \nu_{0},
$$

where $h_{F}\left(\nu_{0}\right)$ denotes the entropy of $\left(F, \nu_{0}\right)$. Let $\xi=(1 / q) \sum_{i=0}^{q-1}\left(f^{i}\right)_{*} \nu_{0}$, which is $f$-invariant. It follows from Lemma $3.2(\mathrm{c})$ that $|\xi(\varphi)-\alpha| \leq \sqrt{\varepsilon}$ since $\nu_{0}$ is supported on $H_{q}(\mathcal{K})$. Then by the definition of $\sigma$ in (12), (16) (17) yield

$$
\begin{aligned}
\log \sum_{i=1}^{\# \mathcal{K}}\left|K_{i}\right|^{\sigma} & \leq h_{F}\left(\nu_{0}\right)-\sigma \int \log |D F| d \nu_{0}+\sigma \log \kappa \\
& =q(h(\xi)-\sigma \lambda(\xi))+\sigma \log \kappa \leq-q \varepsilon^{\frac{1}{3}} \lambda(\xi)+\sigma \log \kappa .
\end{aligned}
$$

Lemma $3.2(b)$ gives

$$
\log \sum_{A \in \mathcal{A}_{n}}|A|^{\sigma} \leq 4 \sqrt{\varepsilon} \sigma n+\log \sum_{i=1}^{\# \mathcal{K}}\left|K_{i}\right|^{\sigma} .
$$

Since $q \geq(1-\varepsilon) n$ and $\lambda(\xi) \geq \lambda_{\text {inf }}>0$ we have

$$
\begin{aligned}
\log \sum_{A \in \mathcal{A}_{n}}|A|^{\sigma} & \leq 4 \sqrt{\varepsilon} \sigma n-q \varepsilon^{\frac{1}{3}} \lambda(\xi)+\sigma \log \kappa \\
& \leq\left(4 \sqrt{\varepsilon} \sigma-(1-\varepsilon) \varepsilon^{\frac{1}{3}} \lambda_{\text {inf }}\right) n+\sigma \log \kappa \leq-\varepsilon^{\frac{1}{3}} \lambda_{\inf } n / 2,
\end{aligned}
$$

where the last inequality holds for sufficiently large $n$. It follows that $\sum_{A \in \mathcal{A}_{n}}|A|^{\sigma}$ has a negative growth rate as $n$ increases. In addition, the above inequality implies that the diameters of the elements of $\mathcal{A}_{n}$ decrease uniformly as $n$ increases. Therefore the Hausdorff $\sigma$-measure of $\Gamma_{k}$ is zero and so $\operatorname{dim}_{H} \Gamma_{k} \leq \sigma$. This completes the proof of Proposition 3.1.

\section{Lower estimate AND CONTINUity of Birkhoff SPECTRUm}

In this section we estimate $B_{\varphi}(\alpha)$ from below, and finish the proof of the formula in Theorem A. We then use this formula to prove the continuity of the Birkhoff spectrum.

4.1. Lower estimate of the Birkhoff spectrum. To estimate $B_{\varphi}(\alpha)$ from below we will construct a sufficiently large set of points for which the time averages of $\varphi$ are precisely equal to $\alpha$. Let $\mathcal{M}_{f}^{e}$ denote the set of ergodic elements of $\mathcal{M}_{f}$. 
Proposition 4.1. Let $\varphi \in C(X)$ and $\alpha \in\left[c_{\varphi}, d_{\varphi}\right]$. Let $\left\{\mu_{i}\right\}_{i}$ be a sequence in $\mathcal{M}_{f}^{e}$ such that $\left|\mu_{i}(\varphi)-\alpha\right|<1 / i$ and $h\left(\mu_{i}\right) / \lambda\left(\mu_{i}\right)$ converges as $i \rightarrow \infty$. There exists a closed set $\Gamma \subset K_{\varphi}(\alpha)$ such that

$$
\operatorname{dim}_{H}(\Gamma) \geq \lim _{i \rightarrow \infty} \frac{h\left(\mu_{i}\right)}{\lambda\left(\mu_{i}\right)}
$$

It then follows that

$$
B_{\varphi}(\alpha) \geq \lim _{\varepsilon \rightarrow 0} \sup \left\{\frac{h(\mu)}{\lambda(\mu)}: \mu \in \mathcal{M}_{f}^{e},|\mu(\varphi)-\alpha|<\varepsilon\right\} .
$$

To finish, it is left to show that the supremum of the right-hand-side of (18) may be taken over all invariant probability measures which are not necessarily ergodic.

Using (A4) and a one-dimensional version of Katok's theorem [18], for any $\mu \in \mathcal{M}_{f}$ and $\varepsilon>0$ one can find $\nu \in \mathcal{M}_{f}^{e}$ such that: $|\mu(\varphi)-\nu(\varphi)| \leq \varepsilon ; h(\nu) \geq h(\mu)-\varepsilon ; \lambda(\nu) \leq \lambda(\mu)+\varepsilon$. Since $0<\lambda_{\text {inf }} \leq \lambda(\mu) \leq \log 4$ and $h(\nu) \leq \log 2$ we have

$$
\frac{h(\nu)}{\lambda(\nu)} \geq \frac{h(\mu)-\varepsilon}{\lambda(\mu)+\varepsilon}=\frac{h(\mu)}{\lambda(\mu)}-\frac{\varepsilon(h(\mu)+\lambda(\mu))}{\lambda(\mu)(\lambda(\mu)+\varepsilon)} \geq \frac{h(\mu)}{\lambda(\mu)}-\frac{3 \varepsilon \log 2}{\lambda_{\text {inf }}^{2}},
$$

and therefore

$$
\sup \left\{\frac{h(\nu)}{\lambda(\nu)}: \nu \in \mathcal{M}_{f}^{e},|\nu(\varphi)-\alpha|<2 \varepsilon\right\} \geq \sup \left\{\frac{h(\mu)}{\lambda(\mu)}: \mu \in \mathcal{M}_{f},|\mu(\varphi)-\alpha|<\varepsilon\right\}-\frac{3 \varepsilon \log 2}{\lambda_{\text {inf }}^{2}} .
$$

Letting $\varepsilon \rightarrow 0$ and then using (18) we obtain

$$
B_{\varphi}(\alpha) \geq \lim _{\varepsilon \rightarrow 0} \sup \left\{\frac{h(\mu)}{\lambda(\mu)}: \mu \in \mathcal{M}_{f},|\mu(\varphi)-\alpha|<\varepsilon\right\} .
$$

From this and the upper estimate in Sect.3 we obtain the formula in Theorem A.

Proof of Proposition 4.1. If $h\left(\mu_{i}\right) \rightarrow 0$ then there is nothing to prove since $\lambda\left(\mu_{i}\right) \geq \lambda_{\text {inf }}>0$. So we may assume $h\left(\mu_{i}\right)>0$ for each $i$. By a result of [18], for any ergodic measure with positive entropy one can construct a horseshoe and use it to approximate its entropy, Lyapunov exponent and the integral of a continuous function. Namely, for each $i$ there exist $\beta_{i}>0$, a closed interval $L_{i}$ and a family $\Omega_{i}$ of pairwise disjoint closed intervals in the interior of $L_{i}$ such that:

(i) for each $I \in \Omega_{i}, f^{\beta_{i}} I=L_{i}$;

(ii) for any $x \in \bigcup_{I \in \Omega_{i}} I$ and $\psi \in\{\varphi, \log |D f|\},\left|\left(1 / \beta_{i}\right) S_{\beta_{i}} \psi(x)-\mu_{i}(\psi)\right| \leq 1 / i$;

(iii) $\left(1 / \beta_{i}\right) \log \# \Omega_{i} \geq h\left(\mu_{i}\right)-1 / i$.

We construct a family of intervals at smaller and smaller scales which wander around different horseshoes. By (A4), for each $i$ it is possible to choose $\gamma_{i}>0$ and a closed interval $\tilde{L}_{i} \subset L_{i}$ such that $f^{\gamma_{i}}$ sends $\tilde{L}_{i}$ homeomorphically onto $Y$. Choose a sequence $\left\{\kappa_{i}\right\}$ of positive integers inductively as follows. Start with $\kappa_{1}=1$. Given $\kappa_{i-1}$, choose $\kappa_{i}$ to be a large integer which depends on $\beta_{1}, \beta_{2}, \ldots, \beta_{i+1}, \gamma_{1}, \gamma_{2}, \ldots, \gamma_{i-1}, \kappa_{1}, \kappa_{2}, \ldots, \kappa_{i-1}, i, \sup |\varphi|, \alpha$. Requirements among these constants will be made explicit at the end of the proof.

For each $k \geq 1$, let $n=n(k), s=s(k)$ be integers such that

$$
k=\kappa_{1}+\kappa_{2}+\cdots+\kappa_{n}+s \text { and } 0 \leq s<\kappa_{n+1} .
$$


Let

$$
\Omega^{(k)}=\underbrace{\Omega_{1} \times \cdots \times \Omega_{1}}_{\kappa_{1}} \times \underbrace{\Omega_{2} \times \cdots \times \Omega_{2}}_{\kappa_{2}} \times \cdots \times \underbrace{\Omega_{n} \times \cdots \times \Omega_{n}}_{\kappa_{n}} \times \underbrace{\Omega_{n+1} \times \cdots \times \Omega_{n+1}}_{s} .
$$

Elements of $\Omega^{(k)}$ are denoted by $\left(I_{1}, \ldots, I_{k}\right)$, i.e., $I_{1} \in \Omega_{1}, \ldots, I_{\kappa_{1}} \in \Omega_{1}, I_{\kappa_{1}+1} \in \Omega_{2}$, and so on.

For each $k \geq 1$ and $\left(I_{1}, \ldots, I_{k}\right) \in \Omega^{(k)}$ we associate a closed interval $\left[I_{1}, \ldots, I_{k}\right]$ inductively as follows. Observe that $\Omega^{(1)}=\Omega_{1}$. For each $I \in \Omega^{(1)}$, define $[I]=I$. Given $k \geq 1$, $\left(I_{1}, \ldots, I_{k}\right) \in \Omega^{(k)},\left[I_{1}, \ldots, I_{k}\right],\left(I_{1}, \ldots, I_{k}, I_{k+1}\right) \in \Omega^{(k+1)}$, define $\left[I_{1}, \ldots, I_{k}, I_{k+1}\right] \subset\left[I_{1}, \ldots, I_{k}\right]$ by

$$
\left[I_{1}, \ldots, I_{k}, I_{k+1}\right]=\left\{\begin{array}{l}
\left(f^{t} \mid\left[I_{1}, \ldots, I_{k}\right]\right)^{-1} I_{k+1} \text { if } s<\kappa_{n+1}-1 \\
\left(f^{t} \mid\left[I_{1}, \ldots, I_{k}\right]\right)^{-1}\left(\left(f^{\beta_{n+1}} \mid I_{k+1}\right)^{-1} \tilde{L}_{n+1}\right) \text { if } s=\kappa_{n+1}-1
\end{array}\right.
$$

where $t=t(k)$ is defined by

$$
t=\beta_{1} \kappa_{1}+\gamma_{1}+\beta_{2} \kappa_{2}+\gamma_{2}+\cdots+\beta_{n} \kappa_{n}+\gamma_{n}+\beta_{n+1} s
$$

Set

$$
\mathcal{F}^{(k)}=\left\{\left[I_{1}, \ldots, I_{k}\right]:\left(I_{1}, \ldots, I_{k}\right) \in \Omega^{(k)}\right\}
$$

This is a collection of pairwise disjoint closed intervals with the following properties: if $s(k)>$ 0 , then $f^{t(k)}\left[I_{1}, \ldots, I_{k}\right]=L_{n(k)+1}$; if $s(k)=0$, then $f^{t(k)}$ sends $\left[I_{1}, \ldots, I_{k}\right]$ homeomorphically onto $Y$. Observe that $\left\{\bigcup_{I \in \mathcal{F}^{(k)}} I\right\}_{k}$ is a nested sequence of closed sets. Define

$$
\Gamma=\bigcap_{k=1}^{\infty} \bigcup_{I \in \mathcal{F}(k)} I
$$

Points in $\Gamma$ continue traveling from one horseshoe to the next generated by $\Omega_{k}, k \geq 1$. For the choice of $\left\{\kappa_{i}\right\}$ we will request

$$
\kappa_{i} \gg \max \left\{\beta_{1}, \beta_{2}, \ldots, \beta_{i+1}, \gamma_{1}, \gamma_{2}, \ldots, \gamma_{i-1}, \kappa_{1}, \kappa_{2}, \ldots, \kappa_{i-1}\right\}
$$

Then, generic finite orbits of $\Gamma$ spend most of their times near the last or the second last horseshoes, and gain time averages in this duration. As a result, the time averages along the finite orbits become nearly $\alpha$. In fact, the following holds.

Lemma 4.2. $\Gamma \subset K_{\varphi}(\alpha)$.

Proof. Let $x \in \Gamma$. For a large integer $q$ let $k \geq 1$ be the maximal such that $t(k) \leq q$. Then $q-t(k) \leq \beta_{n(k)+1}$. Splitting the time interval $[0, q-1]$ is a concatenation of the duration around horseshoes and the transition between horseshoes, and then applying (ii) to each of 
the corresponding orbit segments we have

$$
\begin{aligned}
\left|S_{q} \varphi(x)-q \alpha\right| \leq & \sum_{j=0}^{\kappa_{1}-1}\left|S_{\beta_{1}} \varphi\left(f^{\beta_{1} j} x\right)-\beta_{1} \alpha\right|+\left|S_{\gamma_{1}} \varphi\left(f^{\kappa_{1} \beta_{1}} x\right)-\gamma_{1} \alpha\right| \\
& +\sum_{j=0}^{\kappa_{2}-1}\left|S_{\beta_{2}} \varphi\left(f^{\beta_{1} \kappa_{1}+\gamma_{1}+\beta_{2} j} x\right)-\beta_{2} \alpha\right|+\left|S_{\gamma_{2}} \varphi\left(f^{\beta_{1} \kappa_{1}+\gamma_{1}+\beta_{2} \kappa_{2}} x\right)-\gamma_{2} \alpha\right|+\cdots \\
& +\sum_{j=0}^{\kappa_{n}-1}\left|S_{\beta_{n}} \varphi\left(f^{\sum_{i=1}^{n-1}\left(\beta_{i} \kappa_{i}+\gamma_{i}\right)+\beta_{n} j} x\right)-\beta_{n} \alpha\right|+\left|S_{\gamma_{n}} \varphi\left(f^{\sum_{i=1}^{n} \beta_{i} \kappa_{i}+\sum_{i=1}^{n-1} \gamma_{i}} x\right)-\gamma_{n} \alpha\right| \\
& +\sum_{j=0}^{s-1}\left|S_{\beta_{n+1}} \varphi\left(f^{\sum_{i=1}^{n}\left(\beta_{i} \kappa_{i}+\gamma_{i}\right)+\beta_{n+1} j} x\right)-\beta_{n+1} \alpha\right| \\
& +\left|S_{q-t} \varphi\left(f^{t} x\right)-(q-t) \alpha\right| .
\end{aligned}
$$

Using (ii) and the fact that $x$ is contained in an element of $\mathcal{F}^{(k)}$, for every $2 \leq \ell \leq n$ we have

$$
\begin{aligned}
\sum_{j=0}^{\kappa_{\ell}-1}\left|S_{\beta_{\ell}} \varphi\left(f^{\sum_{i=1}^{\ell-1}\left(\beta_{i} \kappa_{i}+\gamma_{i}\right)+\beta_{\ell} j} x\right)-\beta_{\ell} \alpha\right| \leq & \left|S_{\beta_{\ell}} \varphi\left(f^{\sum_{i=1}^{\ell-1}\left(\beta_{i} \kappa_{i}+\gamma_{i}\right)+\beta_{\ell} j} x\right)-\beta_{\ell} \mu_{\ell}(\varphi)\right| \\
& +\left|\beta_{\ell} \mu_{\ell}(\varphi)-\beta_{\ell} \alpha\right| \leq \frac{2 \beta_{\ell}}{\ell}
\end{aligned}
$$

Summing these and other reminder terms we get

$$
\begin{aligned}
\left|S_{q} \varphi(x)-q \alpha\right| & \leq \sum_{i=1}^{n} \gamma_{i}(\sup |\varphi|+\alpha)+\sum_{i=1}^{n} \frac{2 \beta_{i} \kappa_{i}}{i}+\frac{2 \beta_{n+1} s}{n+1}+(q-t)(\sup |\varphi|+\alpha) \\
& \leq \frac{3 \beta_{n} \kappa_{n}}{n}+\frac{2 \beta_{n+1} s}{n+1} \leq \frac{5 q}{n}
\end{aligned}
$$

where $\kappa_{n}$ is chosen sufficiently large so that the second inequality holds. Since $n \rightarrow \infty$ as $q \rightarrow \infty, x \in K_{\varphi}(\alpha)$ follows.

For each $I \in \mathcal{F}^{(k)}$ choose a point $x_{I} \in I \cap \Gamma$ and define an atomic probability measure $\nu_{k}$ uniformly distributed on the set $\left\{x_{I}: I \in \mathcal{F}^{(k)}\right\}$. Pick an accumulation point of the sequence $\left\{\nu_{k}\right\}$ and denote it by $\nu$. Since $\Gamma$ is closed we have $\nu(\Gamma)=1$. For $x \in X$ and $\rho>0$, let $D_{\rho}(x)=\{y:|x-y| \leq \rho\}$. the inequality in Proposition 4.1 follows from [36, Proposition 2.1] and the next

Lemma 4.3. For any $x \in \Gamma$ we have

$$
\varliminf_{\rho \rightarrow 0} \frac{\log \nu D_{\rho}(x)}{\log \rho} \geq \lim _{i \rightarrow \infty} \frac{h\left(\mu_{i}\right)}{\lambda\left(\mu_{i}\right)} .
$$

Proof. Consider the set of pairs $(n, s)$ of integers such that $n \geq 0$ and $0 \leq s<\kappa_{n+1}$. We introduce an order in this set as follows: $\left(n_{1}, s_{1}\right)<\left(n_{2}, s_{2}\right)$ if $n_{1}<n_{2}$ or $n_{1}=n_{2}$ and $s_{1}<s_{2}$. For a pair $(n, s)$ in this set, let

$$
a_{n, s}=\exp \left[-\beta_{n} \kappa_{n}\left(\lambda\left(\mu_{n}\right)+\frac{2}{n}\right)-\beta_{n+1} s\left(\lambda\left(\mu_{n+1}\right)+\frac{1}{n+1}\right)\right] .
$$


Using (19) it is easy to show that $a_{n+1,0}<a_{n, \kappa_{n+1}-1}$. Hence the sequence $\left\{a_{n, s}\right\}$ is monotone decreasing. Then for given small $\rho>0$ one can choose $k$ such that $a_{n(k), s(k)}<\rho \leq a_{n(k-1), s(k-1)}$.

Let $I \in \mathcal{F}^{(k)}$. We have $\nu(\partial I)=0$, and for every $q \geq k$,

$$
\nu_{q}(I)=\frac{\#\left\{J \in \mathcal{F}^{(q)}: J \subset I\right\}}{\# \mathcal{F}^{(q)}}=\frac{1}{\# \mathcal{F}^{(k)}} .
$$

Hence

$$
\nu(I)=\lim _{q \rightarrow \infty} \nu_{q}(I)=\frac{1}{\# \mathcal{F}^{(k)}} .
$$

Using (ii) for $\psi=\log |D f|$ and (19), for all $x \in I$ we have

$$
\left|D f^{t}(x)\right| \leq \exp \left[\beta_{n} \kappa_{n}\left(\lambda\left(\mu_{n}\right)+\frac{2}{n}\right)+\beta_{n+1} s\left(\lambda\left(\mu_{n+1}\right)+\frac{2}{n}\right)\right] .
$$

Since $f^{t} I \subset X$, the Mean Value Theorem gives

$$
|I| \geq \frac{1}{2} \exp \left[-\beta_{n} \kappa_{n}\left(\lambda\left(\mu_{n}\right)+\frac{2}{n}\right)-\beta_{n+1} s\left(\lambda\left(\mu_{n+1}\right)+\frac{2}{n}\right)\right] .
$$

Hence, for any $x \in \Gamma, D_{\rho}(x)$ intersects at most $2 \exp \left[\beta_{n+1} s(2 / n-1 /(n+1))\right]$-number of elements of $\mathcal{F}^{(k)}$. Using (iii) we have

$$
\# \mathcal{F}^{(k)} \geq\left(\# \Omega_{n}\right)^{\kappa_{n}} \cdot\left(\# \Omega_{n+1}\right)^{s} \geq \exp \left[\beta_{n} \kappa_{n}\left(h\left(\mu_{n}\right)-\frac{1}{n}\right)+\beta_{n+1} s\left(h\left(\mu_{n+1}\right)-\frac{1}{n+1}\right)\right],
$$

and therefore

$$
\begin{aligned}
\nu D_{\rho}(x) & \leq \frac{2}{\# \mathcal{F}^{(k)}} \exp \left[\beta_{n+1} s\left(\frac{2}{n}-\frac{1}{n+1}\right)\right] \\
& \leq 2 \exp \left[-\beta_{n} \kappa_{n}\left(h\left(\mu_{n}\right)-\frac{1}{n}\right)-\beta_{n+1} s\left(h\left(\mu_{n+1}\right)-\frac{2}{n}\right)\right] .
\end{aligned}
$$

This yields

$$
\frac{\log \nu D_{\rho}(x)}{\log \rho} \geq \frac{\beta_{n} \kappa_{n}\left(h\left(\mu_{n}\right)-1 / n\right)+\beta_{n+1} s\left(h\left(\mu_{n+1}\right)-2 / n\right)}{\beta_{n} \kappa_{n}\left(\lambda\left(\mu_{n}\right)+2 / n\right)+\beta_{n+1} s\left(\lambda\left(\mu_{n+1}\right)+1 /(n+1)\right)}+\frac{\log 2}{\log \rho} .
$$

The desired inequality holds since $n \rightarrow \infty$ as $\rho \rightarrow 0$.

4.2. Continuity of the Birkhoff spectrum. From the formula in Theorem A, the spectrum is upper semi-continuous. We argue by contradiction assuming that the spectrum is not lower semi-continuous at a point $\alpha_{0} \in\left[c_{\varphi}, d_{\varphi}\right]$. Then it is possible to choose $\epsilon_{0}>0$ and a monotone sequence $\left\{\alpha_{n}\right\}$ such that $\alpha_{n} \rightarrow \alpha_{0}$ and

$$
B_{\varphi}\left(\alpha_{n}\right) \leq B_{\varphi}\left(\alpha_{0}\right)-\epsilon_{0}
$$

Let us suppose that $\left\{\alpha_{n}\right\}$ is monotone increasing. Take $\mu_{c} \in \mathcal{M}_{f}$ with $\mu_{c}(\varphi)=c_{\varphi}$. The formula in Theorem A allows us to choose a sequence $\left\{\mu_{k}\right\}$ in $\mathcal{M}_{f}$ such that $h\left(\mu_{k}\right) / \lambda\left(\mu_{k}\right) \geq$ $B_{\varphi}\left(\alpha_{0}\right)-\epsilon_{0} / 4$ and $\mu_{k}(\varphi) \rightarrow \alpha_{0}$. Choose a subsequence $\left\{\mu_{k(n)}\right\}$ such that $\alpha_{n} \leq \mu_{k(n)}(\varphi)$. For each $n$ choose $0 \leq t_{n} \leq 1$ such that $\left(1-t_{n}\right) \mu_{c}(\varphi)+t_{n} \mu_{k(n)}(\varphi)=\alpha_{n}$, and define $\nu_{n}=$ $\left(1-t_{n}\right) \mu_{c}+t_{n} \mu_{k(n)}$. For all large $n$ we have

$$
B_{\varphi}\left(\alpha_{n}\right)=B_{\varphi}\left(\left(1-t_{n}\right) \mu_{c}(\varphi)+t_{n} \mu_{k(n)}(\varphi)\right) \geq \frac{h\left(\nu_{n}\right)}{\lambda\left(\nu_{n}\right)} \geq B_{\varphi}\left(\alpha_{0}\right)-\epsilon_{0} / 2 .
$$


The second inequality follows from the linearity of entropies and Lyapunov exponents on measures, $t_{n} \rightarrow 1$ and $\inf _{n} \lambda\left(\mu_{k(n)}\right) \geq \lambda_{\text {inf }}>0$. This yields a contradiction to (21). In the case where $\left\{\alpha_{n}\right\}$ is monotone decreasing, take $\mu_{d} \in \mathcal{M}_{f}$ with $\mu_{d}(\varphi)=d_{\varphi}$ and use it in the place of $\mu_{c}$.

\section{LARGE DEVIATION PRINCIPLE}

In this last section we prove Theorem B. This amounts to proving the next proposition which gives an upper bound of deviation probabilities in terms of the free energies of invariant measures.

Proposition 5.1. Let $d \geq 1, \varphi_{1}, \ldots, \varphi_{d} \in C(X)$ be Lipschitz, and let $\alpha_{1}, \ldots, \alpha_{d} \in \mathbb{R}$. For any $\varepsilon>0$ there exists $n_{0}>0$ such that for every $n \geq n_{0}$ there exists $\eta \in \mathcal{M}_{f}$ such that:

$$
\begin{gathered}
\frac{1}{n} \log \left|\left\{x \in \Lambda: \frac{1}{n} S_{n} \varphi_{j}(x) \geq \alpha_{j}, j=1, \ldots, d\right\}\right| \leq(1-\varepsilon) F(\eta)+4 \sqrt{\varepsilon} \\
\eta\left(\varphi_{j}\right) \geq \alpha_{j}-\sqrt{\varepsilon}, \quad j=1, \ldots, d .
\end{gathered}
$$

We finish the proof of Theorem B assuming the conclusion of the proposition. Recall that $M>0$ is such that $f^{M} \Lambda=Y$. Let $\varepsilon_{0}>0$ be a small constant. For all large $n$ we have

$$
\left\{x \in Y: \frac{1}{n} S_{n} \varphi_{j}(x) \geq \alpha_{j}\right\} \subset f^{M}\left\{x \in \Lambda: \frac{1}{n} S_{n} \varphi_{j}(x) \geq \alpha_{j}-\varepsilon_{0}\right\},
$$

where it is understood that $j$ runs over $\{1,2, \ldots, d\}$. By the Mean Value Theorem,

$$
\left|\left\{x \in Y: \frac{1}{n} S_{n} \varphi_{j}(x) \geq \alpha_{j}\right\}\right| \leq 4^{M} \cdot\left|\left\{x \in \Lambda: \frac{1}{n} S_{n} \varphi_{j}(x) \geq \alpha_{j}-\varepsilon_{0}\right\}\right| .
$$

From this inequality and Proposition 5.1 there exists $\eta \in \mathcal{M}_{f}$ such that $\eta\left(\varphi_{j}\right) \geq \alpha_{j}-\varepsilon_{0}-\sqrt{\varepsilon}$ $(j=1, \ldots, d)$ and

$$
\frac{1}{n} \log \left|\left\{x \in Y: \frac{1}{n} S_{n} \varphi_{j}(x) \geq \alpha_{j}\right\}\right| \leq \frac{M}{n} \log 4+(1-C \varepsilon) F(\eta)+4 \sqrt{\varepsilon} .
$$

Letting $n \rightarrow \infty$, and then $\varepsilon_{0} \rightarrow 0, \varepsilon \rightarrow 0$ we get

$$
\varlimsup_{n \rightarrow \infty} \frac{1}{n} \log \left|\left\{\frac{1}{n} S_{n} \varphi_{j} \geq \alpha_{j}\right\}\right| \leq \lim _{\varepsilon \rightarrow 0} \sup \left\{F(\nu): \nu \in \mathcal{M}_{f}, \nu\left(\varphi_{j}\right) \geq \alpha_{j}-\sqrt{\varepsilon}\right\} .
$$

The lower large deviations bound obtained in [7] gives

$$
\underline{\lim _{n \rightarrow \infty}} \frac{1}{n} \log \left|\left\{\frac{1}{n} S_{n} \varphi_{j}>\alpha_{j}\right\}\right| \geq \sup \left\{F(\nu): \nu \in \mathcal{M}, \nu\left(\varphi_{j}\right)>\alpha_{j}\right\},
$$

where $\sup \emptyset=-\infty$. Theorem B follows from (24) (25) because the weak topology on $\mathcal{M}$ has a countable base generated by open sets of the form $\left\{\nu \in \mathcal{M}: \nu\left(\varphi_{j}\right)>\alpha_{j}, j=1, \ldots, d\right\}$, where $d \geq 1$, each $\varphi_{j} \in C(X)$ is Lipschitz, and $\alpha_{j} \in \mathbb{R}$.

The rest of this section is devoted to the proof of Proposition 5.1. From the towers constructed in Sect.2 we extract horseshoes, and construct invariant measures supported on them with the properties as in the statement of the proposition. 
5.1. Construction of a horseshoe. Define

$$
\mathcal{B}_{n}=\left\{A \in \bigvee_{i=0}^{n-1} \hat{f}^{-i} \mathcal{D}: A \subset \Delta_{0}, \quad \frac{1}{n} S_{n} \varphi_{j}(x) \geq \alpha_{j} \quad j=1, \ldots, d \text { for some } x \in A\right\} .
$$

Observe that

$$
\left|\left\{x \in \Lambda: \frac{1}{n} S_{n} \varphi_{j}(x) \geq \alpha_{j} \quad j=1, \ldots, d\right\}\right| \leq\left|\mathcal{B}_{n}\right|,
$$

where $\left|\mathcal{B}_{n}\right|=\sum_{A \in \mathcal{B}_{n}}|A|$. To estimate this from above we use the next lemma, the proof of which closely follows that of Lemma 3.2 with $\sigma$ replaced by 1 .

Lemma 5.2. For any $\varepsilon>0$ there exists $n^{\prime \prime}>0$ such that if $n \geq n^{\prime \prime}$ and $\mathcal{B}_{n} \neq \emptyset$ then there exist a finite collection $\mathcal{L}$ of pairwise disjoint closed intervals in $\Lambda$ and an integer $r \in$ $[(1-\varepsilon) n,(1+21 \varepsilon / \lambda) n]$ such that:

(a) $\mathcal{L}$ generates a horseshoe for $f^{r}$;

(b) $\sum_{L \in \mathcal{L}}|L| \geq e^{-3 \sqrt{\varepsilon} n}\left|\mathcal{B}_{n}\right|$;

(c) for all $x \in H_{r}(\mathcal{L}),(1 / r) S_{r} \varphi_{j}(x) \geq \alpha_{j}-\sqrt{\varepsilon}, j=1, \ldots, d$.

Proof. For each $B \in \mathcal{B}_{n}$ fix once and for all an interval $\tilde{B}$ and an integer $t=t_{B}$ for which the conclusions of Lemma 2.10 holds. Let $\mathcal{B}_{n}(t)=\left\{B \in \mathcal{B}_{n}: t_{B}=t\right\}$. Then $t_{B} \in[(1-\varepsilon) n,(1+$ $19 \varepsilon / \lambda) n]$. Let $t_{1}$ be a value of $t$ which maximizes $\sum_{B \in \mathcal{B}_{n}(t)}|\tilde{B}|$. Then

$$
\sum_{B \in \mathcal{B}_{n}\left(t_{1}\right)}|\tilde{B}| \geq \frac{1}{(1+20 / \lambda) \varepsilon n} \sum_{B \in \mathcal{B}_{n}}|\tilde{B}| .
$$

For each $B \in \mathcal{B}_{n}\left(t_{1}\right)$ define $L(B)$ to be the preimage of $I^{+}$or $I^{-}$under $f^{t_{1}} \mid \tilde{B}$, according to whether $f^{t_{1}} \tilde{B}=\Lambda^{+}$or $=\Lambda^{-}$. Set $\mathcal{L}=\left\{L(B): B \in \mathcal{B}_{n}\left(t_{1}\right)\right\}$ and $r=t_{1}+u$. The bounds on $r$ hold for sufficiently large $n$. (27) and Lemma 2.10 implies

$$
\sum_{L \in \mathcal{L}}|L| \geq e^{-3 \sqrt{\varepsilon} n}\left|\mathcal{B}_{n}\right|
$$

To prove (c), for each $B \in \mathcal{B}_{n}\left(t_{1}\right)$ pick $x_{B} \in B$ such that $S_{n} \varphi\left(x_{B}\right) \geq \alpha_{j} n$ for $j=1, \ldots, d$. We have

$$
S_{r} \varphi_{j}\left(x_{B}\right) \geq S_{n} \varphi_{j}\left(x_{B}\right)-\sup \left|\varphi_{j}\right| \cdot|r-n| \geq \alpha_{j} n-(21 \varepsilon / \lambda) n \geq\left(\alpha_{j}-\sqrt{\varepsilon} / 2\right) r .
$$

By Sublemma 3.3, for any $x \in B$ we have $\left|S_{t_{1}} \varphi_{j}\left(x_{B}\right)-S_{t_{1}} \varphi_{j}(x)\right| \leq \operatorname{Lip}\left(\varphi_{j}\right) \cdot C \delta^{-1}$, and thus

$$
\left|S_{r} \varphi_{j}\left(x_{B}\right)-S_{r} \varphi_{j}(x)\right| \leq \operatorname{Lip}\left(\varphi_{j}\right) \cdot C \delta^{-1}+2 \sup \left|\varphi_{j}\right| \cdot\left(r-t_{1}\right) \leq \sqrt{\varepsilon} r / 2 .
$$

(28) (29) yield $S_{r} \varphi_{j}(x) \geq\left(\alpha_{j}-\sqrt{\varepsilon}\right) r$.

5.2. Construction of a measure on the horseshoe. We construct a measure $\eta$ for which (22) (23) hold. For sufficiently large $n$ with $\mathcal{B}_{n} \neq \emptyset$, choose a finite collection $\mathcal{L}$ of pairwise disjoint closed intervals in $\Lambda$ and a positive integer $r$ for which the conclusions of Lemma 5.2 hold. Set $G=f^{r}$. The argument in Sect 3.2 shows that there exists a $G$-invariant probability measure $\nu_{\infty}$ supported on $H_{r}(\mathcal{L})$ satisfying

$$
h_{G}\left(\nu_{\infty}\right)-\int \log |D G| d \nu_{\infty} \geq \log \sum_{L \in \mathcal{L}}|L|-\log \kappa,
$$


where $h_{G}\left(\nu_{\infty}\right)$ denotes the entropy of $\left(G, \nu_{\infty}\right)$. Define $\eta \in \mathcal{M}_{f}$ by $\eta=(1 / r) \sum_{i=0}^{r-1}\left(f^{i}\right)_{*} \nu_{\infty}$. From Lemma 5.2 (c) it follows that $\eta\left(\varphi_{j}\right) \geq \alpha_{j}-\sqrt{\varepsilon}$, and (23) holds. Since $F(\eta) \leq 0$ and $r \geq(1-\varepsilon) n$, using Lemma 5.2 (b) we have

$$
\begin{aligned}
n \cdot F(\eta) & \geq \frac{r}{1-\varepsilon} F(\eta)=\frac{1}{1-\varepsilon}\left(h_{G}\left(\nu_{\infty}\right)-\int \log |D G| d \nu_{\infty}\right) \\
& \geq \frac{1}{1-\varepsilon}\left(\log \sum_{L \in \mathcal{L}}|L|-\log \kappa\right) \geq \frac{1}{1-\varepsilon}\left(\log \left|\mathcal{B}_{n}\right|-4 \sqrt{\varepsilon} n\right) .
\end{aligned}
$$

Rearranging this and using (26) yields

$$
\frac{1}{n} \log \left|\left\{x \in \Lambda: \frac{1}{n} S_{n} \varphi_{j}(x) \geq \alpha_{j} \quad j=1, \ldots, d\right\}\right| \leq \frac{1}{n} \log \left|\mathcal{B}_{n}\right| \leq(1-\varepsilon) F(\eta)+4 \sqrt{\varepsilon} .
$$

Hence (22) holds.

Acknowledgments. We thank anonymous referees for very useful comments. The firstnamed author is partially supported by the Grant-in-Aid for Scientific Research (C) of the JSPS, Grant No. 24540212. The second-named author is partially supported by the Grantin-Aid for Young Scientists (B) of the JSPS, Grant No. 23740121.

\section{REFERENCES}

1. L. Barreira and J. Schmeling. Sets of "non-typical" points have full Hausdorff dimension and full topological entropy. Israel J. Math. 116 (2000), 29-70.

2. M. Benedicks and L. Carleson. On iterations of $1-a x^{2}$ on $(-1,1)$. Ann. Math. 122 (1985), 1-25.

3. M. Benedicks and L. Carleson. The dynamics of the Hénon map. Ann. Math. 133 (1991), 73-169.

4. M. Benedicks and L.-S. Young. Absolutely continuous invariant measures and random perturbations for certain one-dimensional maps. Ergod. Th. \& Dynam. Sys. 12 (1992), 13-37.

5. H. Bruin and G. Keller. Equilibrium states for S-unimodal maps. Ergod. Th. \& Dynam. Sys. 18 (1998), 765-789.

6. Y. M. Chung. Birkhoff spectra for one-dimensional maps with some hyperbolicity. Stochastics and Dynamics 10 (2010), 53-75.

7. Y. M. Chung. Large deviations on Markov towers. Nonlinearity 24 (2011), 1229-1252.

8. Y. M. Chung and H. Takahasi. Large deviation principle for Benedicks-Carleson quadratic maps: Comm. Math. Phys. 315 (2012) 803-826

9. V. Climenhaga. Bowen's equation in the non-uniform setting: Ergod. Th. \& Dynam. Sys. 31 (2011), $1163-1182$.

10. H. Comman and J. Rivera-Letelier. Large deviation principles for non-uniformly hyperbolic rational maps. Ergod. Th. \& Dynam. Sys. 31 (2011), 321-349.

11. A. Dembo and O. Zeitouni. Large Deviations Techniques and Applications. 2nd edn, Applications of Mathematics 38, Springer, New York, 1998.

12. K. Gelfert, F. Przytycki and M. Rams. On the Lyapunov spectrum for rational maps. Math. Ann. 348 (2010), 965-1004.

13. P. Grassberger, R. Badii, and A. Politi. Scaling laws for invariant measures on hyperbolic and nonhyperbolic attractors. J. Stat. Phys. 51 (1988), 135-178.

14. T. C. Hasley, M. Jensen, L. Kadanoff, I. Procaccia and B. Shraiman. Fractal measures and their singularities: the characterization of strange sets. Phys. Rev. A 33:2 (1986), 1141-1151.

15. F. Hofbauer. Local dimension for piecewise monotone maps on the interval. Ergod. Th. \& Dynam. Sys. 15 (1995), 1119-1142.

16. G. Iommi and M. Todd. Dimension theory for multimodal maps. Ann. Henri Poincaré 12 (2011), 591-620.

17. M. Jakobson. Absolutely continuous invariant measures for one-parameter families of one-dimensional maps. Comm. Math. Phys. 81 (1981), 39-88. 
18. A. Katok. Lyapunov exponents, entropy and periodic orbits for diffeomorphisms. Publ. Math. Inst. Hautes Étud. Sci. 51 (1980), 137-173.

19. G. Keller and T. Nowicki. Spectral theory, zeta functions and the distribution of periodic points for Collet-Eckmann maps. Comm. Math. Phys. 149 (1992), 31-69.

20. Y. Kifer. Large deviations in dynamical systems and stochastic processes. Trans. Amer. Math. Soc. 321 (1990), 505-524.

21. F. Ledrappier. Some properties of absolutely continuous invariant measures of an interval. Ergod. Th. \& Dynam. Sys. 1 (1981), 77-93.

22. F. Ledrappier. Some relations between dimension and Lyapunov exponents. Comm. Math. Phys. 81 (1981), 229-238.

23. W. de Melo and S. van Strien. One-dimensional dynamics. Springer, Berlin, Heidelberg, New York, 1993.

24. K. Nakaishi. Multifractal formalism for some parabolic maps. Ergod. Th. \& Dynam. Sys. 20 (2000), 843-857.

25. T. Nowicki and D. Sands. Non-uniform hyperbolicity and universal bounds for S-unimodal maps. Invent. Math. 132 (1998), 633-680.

26. L. Olsen. Multifractal analysis of divergence points of deformed measure theoretical Birkhoff averages. $J$. Math. Pures Appl. 82 (2003), 1591-1649.

27. S. Orey and S. Pelikan. Deviations of trajectory averages and the defect in Pesin's formula for Anosov diffeomorphisms. Trans. Amer. Math. Soc. 315 (1989), 741-753.

28. Y. Pesin. Dimension Theory in Dynamical Systems, Univ. of Chicago Press, Chicago, 1997.

29. Y. Pesin and H. Weiss. A multifracatal analysis of equilibrium measures for conformal expanding maps and Moran-like geometric constructions. J. Stat. Phys. 86 (1997), 233-275.

30. Y. Pesin and H. Weiss. The multifractal analysis of Birkhoff averages and large deviations, in Global Analysis of Dynamical Systems, eds. H. W. Broer, B. Krauskopf and G. Vegter, Inst. Phys., Bristol (2001), pp. 419-431.

31. F. Przytycki and J. Rivera-Letelier. Nice inducing schemes and the thermodynamics of rational maps. Comm. Math. Phys. 70 (2011), 661-707.

32. L. Rey-Bellet and L.-S. Young. Large deviations in non-uniformly hyperbolic dynamical systems. Ergod. Th. \& Dynam. Sys. 28 (2008), 587-612.

33. D. Ruelle. An inequality for the entropy of differentiable maps. Bol. Soc. Mat. Bras. 9 (1978), 83-87.

34. P. Walters. An introduction to ergodic theory. Graduate Texts in Mathematics 79, Springer, New York, 1982.

35. H. Weiss. The Lyapunov spectrum for conformal expanding maps and Axiom A surface diffeomorphisms. J. Stat. Phys. 95 (1999), 615-632.

36. L.-S. Young. Dimension, entropy and Lyapunov exponents. Ergod. Th. \& Dynam. Sys. 2 (1982), 109-124.

37. L.-S. Young. Decay of correlations of certain quadratic maps. Comm. Math. Phys. 146 (1992), 123-138. 